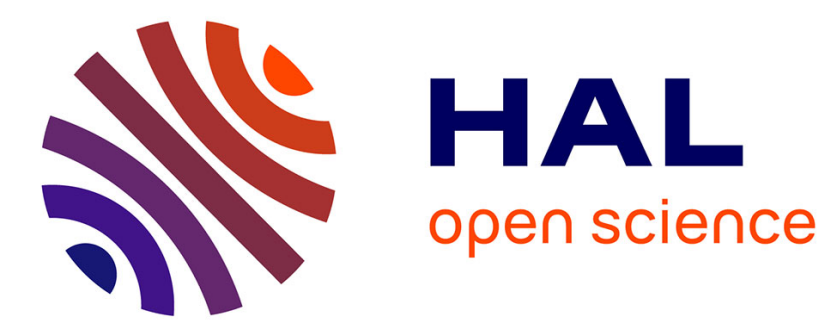

\title{
Improvements for a Fully Consistent Description of the New Semi-Empirical Vapor Density Model for Pure Compounds
}

\author{
Alexander Kud, Christophe Coquelet
}

\section{- To cite this version:}

Alexander Kud, Christophe Coquelet. Improvements for a Fully Consistent Description of the New Semi-Empirical Vapor Density Model for Pure Compounds. Fluid Phase Equilibria, 2022, 556, pp.113374. 10.1016/j.fluid.2021.113374 . hal-03514283

\section{HAL Id: hal-03514283 \\ https://hal.science/hal-03514283}

Submitted on 28 Jan 2022

HAL is a multi-disciplinary open access archive for the deposit and dissemination of scientific research documents, whether they are published or not. The documents may come from teaching and research institutions in France or abroad, or from public or private research centers.
L'archive ouverte pluridisciplinaire HAL, est destinée au dépôt et à la diffusion de documents scientifiques de niveau recherche, publiés ou non, émanant des établissements d'enseignement et de recherche français ou étrangers, des laboratoires publics ou privés. 
Improvements for a Fully Consistent Description of the New SemiEmpirical Vapor Density Model for Pure Compounds

Alexander Kud ${ }^{\mathrm{a}, *}$, Christophe Coquelet ${ }^{\mathrm{b}, \mathrm{c}}$

${ }^{\star}$ Corresponding author, Germany, E-mail: alexander.e.kud@yandex.com ${ }^{a}$ retired Physical Chemist from Department of Research, BASF SE, Germany bMines ParisTech, PSL University, CTP - Centre of Thermodynamics of Processes, 35 rue Saint Honoré, Fontainebleau Cedex 77305, France 'Université de Toulouse, IMT Mines Albi, UMR CNRS 5302, Centre Rapsodee, Campus Jarlard, 81013 Albi CT Cedex 9, France

E-mail: christophe.coquelet@mines-albi.fr

\section{Abstract}

In chemical engineering, surrogate functions are commonly used in modeling thermodynamic properties of pure compounds because they are often more accurate than equations of state. In 2020, we presented a semi-empirical model describing the vapor density from the reduced triple point temperature to about a reduced temperature of 0.97 with only one temperature-dependent term. However, in many cases the temperature range up to critical temperature is necessary. Therefore, the existing semi-empirical model was extended by another temperature-dependent term. This allows the vapor density to be described from the triple point temperature up to the critical temperature. This model has the additional advantage that it can predict the vapor pressure data from vapor density data. Therefore, this model can also be used for thermodynamic consistency testing. This is not possible with conventionally known surrogate models for describing vapor density. The model was successfully tested using fluoromethane (R41) and difluo-romethane (R32) as examples.

Keywords: vapor density, thermodynamic consistency, fluoromethane (R41), difluoromethane (R32),

\section{Introduction}

In chemical engineering, reactors and apparatuses are often designed by considering the presence of liquid and/or vapor phases. In order to correctly realize the mass balance for both phases, their densities must be correctly calculated. This aspect is valuable for the design of reactor where mass and volume fractions depend on density calculation. In effect, 
an incorrect density prediction does not only influence the calculation results of the Vapor Liquid Equilibrium (VLE) but also the result of a kinetic modeling, if chemical reactions have to be considered.

Because equations of state are not so accurate to calculate state variables at saturation like vapor and liquid densities, surrogate functions are used for modeling. Lists of such functions for pure substances are documented, for example, at the National Institute of Standards and Technology (NIST) [1] and at the Dortmund Data Bank [2]. In general, the equations for vapor density given there are empirical and have some weaknesses due to their lack of physical meanings. In our previous work (Kud et al. [3]), we presented a Semi-empirical Model (SeM) that considers compressibility factor as thermodynamic quantity and also kud et al. [3] give an overview of the currently used surrogate models in Tab. 1, with which the new model is compared. The compressibility factor for vapor describes a graph characteristic of a compound as a function of temperature, and according to Nezbeda [4], it is called a "strong test of precision and correctness". In 2020, an empirical equation ( $Z$ model) has been developed for the compressibility factor of vapor, which represents the physical properties from the triple point to the critical temperature. The concept and ideas that have been considered for the development of the new $Z$ model are the core of the vapor density model (SeM, ref [3]). Also, the vapor density model can be used not only for vapor density description. It can be used, according to Eggimann et al. [5], to check and evaluate the quality of molecular simulations with the transferable potentials for phase equilibria (TraPPE) model and also to check the quality of experimental data. For example, 20 compounds of different structure and polarity can be represented in a "master plot" with one graph, see abstract and Fig. 5 in their publication [5].

The semi-empirical vapor density model was successfully tested for its principal suitability on 17 compounds from different families of mole-cules. These molecules have different structure and polarity.

In many cases, however, it is necessary to calculate the vapor density and thus the compressibility factor from the triple point to the critical temperatures. This work is the continuity of our previous paper [3] and it is an extension of first model from triple point (and below) to the critical point combining pure component vapor pressure and compressibility factor equation at saturation. For this purpose, an additional, tempera-turedependent term is used in the $Z$-model. The thermodynamic inconsisten-cies occurring in the parameterization at the level of the triple point temperature must be eliminated by taking into account the corresponding physical property values. The results are compared with the state of the art. For this purpose, two polar compounds, namely fluoromethane (R41) and difluoromethane (R32) are used as examples. This is the subject of this work. 
The basic idea of the new SeM will be briefly explained on the basis of a schematic representation in figure 1 . The compressibility factor $Z$ for the vapor state combines the two state variables vapor pressure $p$ and vapor density $\varrho$ at the absolute temperature $T$ according to the equation

$Z=\frac{p V}{n \mathcal{R} T}=\frac{p v}{\mathcal{R} T}=\frac{p \mathcal{M}}{\varrho \mathcal{R} T}$,

where $V$ is the volume, $\boldsymbol{n}$ the amount of matter, $v$ the molar volume, $\mathcal{M}$ the molar mass of the compound and $\mathcal{R}$ the universal gas constant. Introducing the reduced state variables for a pure compound according to $\tau=T / T_{c}, \quad \pi=$ $p / p_{c}$ (where $T_{c}$ is the critical temperature, $p_{c}$ the critical pressure) and the constants $\varrho_{c}$ for the critical density and $Z_{c}$ for the critical compressibility factor, we obtain the following equation for the vapor density.

$\varrho=\varrho_{c} Z_{c} \frac{\pi}{\tau Z}$.

The Semi-empirical vapor density model is obtained by substituting a vapor pressure function $f_{p}\left(\tau, \Theta_{p}\right)$ for the reduced vapor pressure $\pi$ and by substituting the empirical $Z_{M}\left(\tau, \Theta_{Z}\right)$ model

$Z_{M}\left(\tau, \Theta_{Z}\right)=Z_{c}+\left(1-Z_{c}\right) \cdot\left\{\begin{array}{cl}{\left[1-\left(\frac{\tau-\Theta_{z 3} \cdot \bar{\tau}_{t p}}{1-\Theta_{z 3} \cdot \bar{t}_{t p}}\right)^{\Theta_{z 1}}\right]^{\Theta_{Z 2}}} & \text { if } \tau_{T p} \leq \tau \leq 0.96 \\ \text { not defined } & \text { if } \tau>1\end{array}\right.$

for the compressibility factor $Z$ of the vapor in equation (2) where $\Theta_{p}$ and $\Theta_{z}$ are the parameter vectors. Thus, the vapor density $\varrho_{M}$ can be calculated according to the model equation $F_{\varrho}\left(\tau, \Theta_{p}, \Theta_{z}\right)$

$\varrho_{M}=F_{Q}\left(\tau, \Theta_{p}, \Theta_{Z}\right)=\varrho_{c} Z_{c} \frac{f_{p}\left(\tau, \Theta_{p}\right)}{\tau Z_{M}\left(\tau, \Theta_{z}\right)}$

The parameterization of equation (4) is based on the measured vapor pressures $\bar{Y}_{p}$ and vapor densities $\bar{Y}_{\varrho}$. Figure 1 illustrates this situation. 


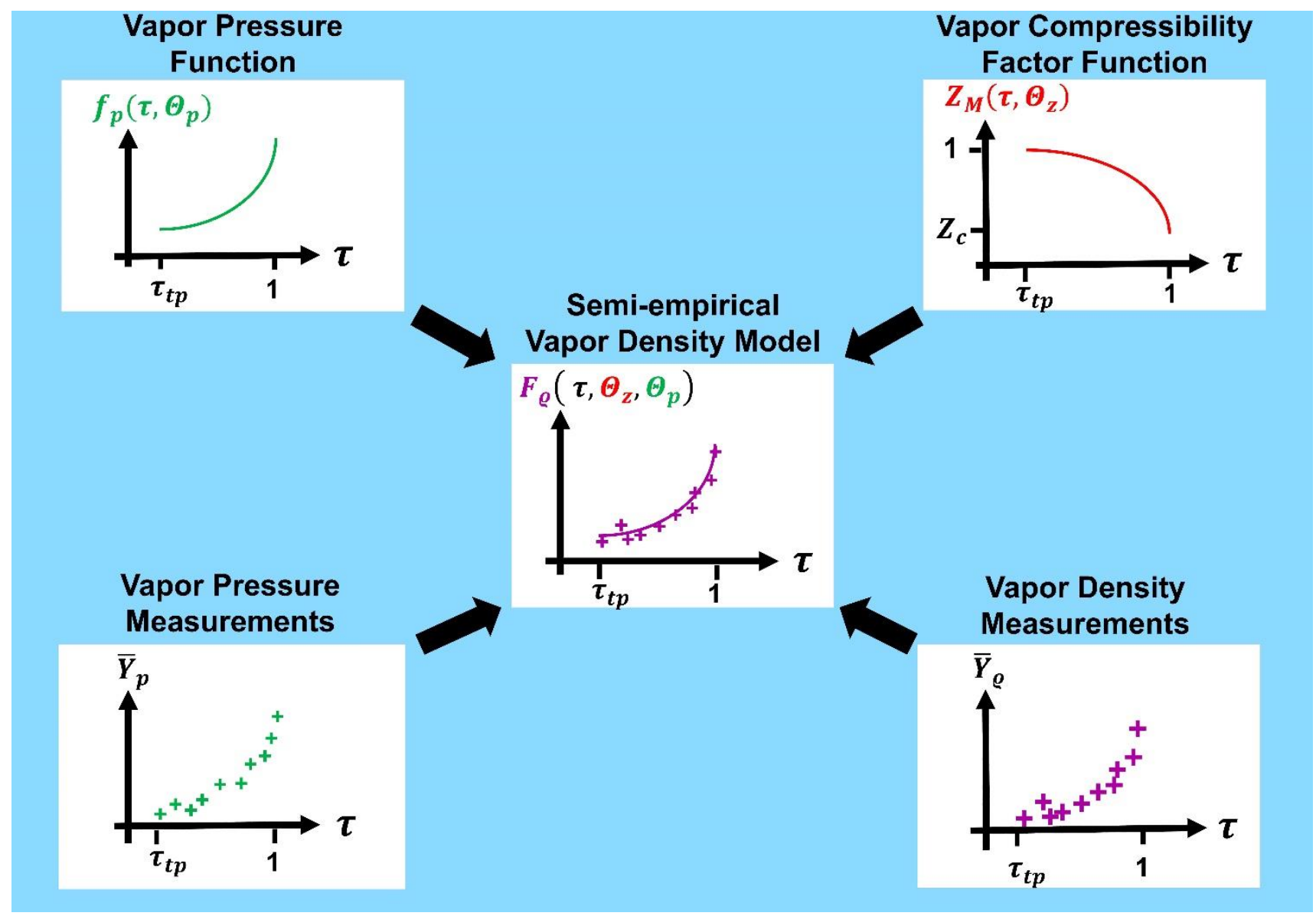

Fig. 1. Schematic representation of the development of the semi-empirical vapor density model for a pure compound as a function of the reduced temperature $\tau$ from reduced triple point temperature $\tau_{T p}$ to the critical temperature $\tau_{c}=1 . f_{p}\left(\tau, \Theta_{p}\right)$ is the vapor pressure function, $Z_{M}\left(\tau, \Theta_{Z}\right)$ is the function for the compressibility factor of the vapor, $F_{Q}\left(\tau, \Theta_{p}, \Theta_{Z}\right)$ is the semi-empirical model equation for the vapor, $\Theta_{p}, \Theta_{z}$ are the model parameters, $\bar{Y}_{p}$, $\bar{Y}_{Q}$ are the experimental data.

At this point, a definition of the vapor state as it is considered in this work should be given in order to avoid misunderstandings with the gas state. The vapor state is always linked to the presence of a liquid and/or solid phase with which it is in phase equilibrium. This means that there is an exchange of substances between the two phases and the density of vapor is constant over time. Thus, there is a so-called Vapor Liquid Equilibrium (VLE) or a Vapor Solid Equilibrium (VSE). The gas state, on the other hand, exists independently of the presence of a liquid or solid state. For the sake of simplicity, all variables and quantities in this paper refer to the vapor state.

2 Theoretical background and computational modeling

The most important equations should be presented before the presentation of our strategy of modeling. The description in the arguments of the equations 
should be as exact as possible, so that the dependencies of the calculation results become clearly recognizable and conscious. Afterwards simplified representations in the arguments are defined. We use the vapor pressure function $F_{p}\left(\tau, T_{c}, p_{c}, \Theta_{p}\right)$ respectively $f_{p}\left(\tau, T_{c}, \Theta_{p}\right)$ for calculating reduced vapor pressure according on the rearranged Riedel equation [6]. $f_{p}\left(\tau, T_{c}, \Theta_{p}\right)$ is a semi-empirical model and is based on the corresponding state principle. This equation with the parameter vector $\Theta_{p}$ is also called DIPPR (Design Institute for Physical Properties) 101 equation and is given by Eqs. (5-6)

$F_{p}\left(\tau, T_{c}, p_{c}, \Theta_{p}\right)=p_{c} \cdot f_{p}\left(\tau, T_{c}, \Theta_{p}\right)$

$f_{p}\left(\tau, T_{c}, \Theta_{p}\right)=\exp \left[\frac{\Theta_{p 1}}{T_{c}}\left(1-\frac{1}{\tau}\right)+\Theta_{p 2} \cdot \ln (\tau)+\Theta_{p 3} \cdot T_{c}^{\Theta_{p 4}}\left(\tau^{\Theta_{p 4}}-1\right)\right]$.

The parameter $\Theta_{p 4}$ is an integer and arbitrarily kept constant to one of the values from 1 to 6 , while the remaining parameters are adjusted. The minimum of the objective function from the six parameter estimates sets the value of the optimal parameter $\Theta_{p 4, o p t}$. Hogge et al. [7] have tested this equation for the temperature range considered in our work here on many compounds of different structure and they found it is a very good equation. Hogge et al. recommend an exponent $\Theta_{p 4, o p t}=2$. Concerning the SeM, a similar situation exists with the number of terms $\mathcal{N}_{z}$ in the model function for the compressibility factor of vapor $Z_{M}$. For reasons of simplified notation in the function arguments, the number of terms shall be included in the parameter vector $\Theta_{z}$, knowing that they are not estimated.

$Z_{M}\left(\tau, Z_{c}, \tau_{t p}, \Theta_{z}\right)=Z_{c}+\left(1-Z_{c}\right) \cdot \begin{cases}{\left[1-\left(\frac{\tau-\Theta_{z 3} \cdot \tau_{t p}}{1-\theta_{z 3} \cdot \tau_{t p}}\right)^{\theta_{z 1}}\right]^{\Theta_{z 2}}} & \text { if } \Theta_{z 4}=\mathcal{N}_{z}=1, \tau_{t p} \leq \tau \leq 0.96 \\ \left\{\begin{array}{l}\Theta_{z 6}\left[1-\left(\frac{\tau-\Theta_{z 3} \cdot \tau_{t p}}{1-\theta_{z 3} \cdot \tau_{t p}}\right)^{\theta_{z 1}}\right]^{\theta_{z 2}}+ \\ \left(1-\Theta_{z 6}\right)\left[1-\left(\frac{\tau-\theta_{z 3} \cdot \tau_{t p}}{1-\theta_{z 3} \cdot \tau_{t p}}\right)^{\theta_{z 4}}\right]^{\theta_{z 5}}\end{array}\right. & \text { if } \Theta_{z 7}=\mathcal{N}_{z}=2, \tau_{t p} \leq \tau \leq 1 \\ \text { not defined } & \text { if } \tau>1\end{cases}$

$\Theta_{z 4}$ and $\Theta_{z 7}$ are the numbers of terms. The model for calculating the vapor density $\varrho_{M}$ or reduced vapor density $\omega$ in reduced state variables is given by Eqs. (8-9)

$\varrho_{M}=F_{\varrho}\left(\tau, T_{c}, \varrho_{c}, Z_{c}, \tau_{t p}, \Theta_{p}, \Theta_{z}\right)=\varrho_{c} \cdot f_{\varrho}\left(\tau, T_{c}, Z_{c}, \tau_{t p}, \Theta_{p}, \Theta_{z}\right)$

$\omega=f_{\varrho}\left(\tau, T_{c}, Z_{c}, \tau_{t p}, \Theta_{p}, \Theta_{z}\right)=Z_{c} \frac{f_{p}\left(\tau, T_{c}, \Theta_{p}\right)}{\tau \cdot Z_{M}\left(\tau, Z_{c}, \tau_{t p}, \Theta_{z}\right)}$.

The critical compressibility factor $Z_{c}$ is calculated according to the known relation $Z_{c}={ }^{\mathcal{M}} p_{c} /\left(\mathcal{R} T_{c} \varrho_{c}\right)$ from the critical properties $T_{c}$, $p_{c}$ and $\varrho_{c}$. For further developments, the following identities (10 to 14 ) are applied due to a simplify notations:

$$
\begin{aligned}
& F_{p}\left(\tau, \Theta_{p}\right) \equiv F_{p}\left(\tau, T_{c}, p_{c}, \Theta_{p}\right) \\
& f_{p}\left(\tau, \Theta_{p}\right) \equiv f_{p}\left(\tau, T_{c}, \Theta_{p}\right) \\
& F_{\varrho}\left(\tau, \Theta_{p}, \Theta_{z}\right) \equiv F_{\varrho}\left(\tau, T_{c}, \varrho_{c}, Z_{c}, \tau_{t p}, \Theta_{p}, \Theta_{z}\right)
\end{aligned}
$$


$f_{\varrho}\left(\tau, \Theta_{p}, \Theta_{z}\right) \equiv f_{\varrho}\left(\tau, T_{c}, Z_{c}, \tau_{t p}, \Theta_{p}, \Theta_{z}\right)$

$Z_{M}\left(\tau, \Theta_{Z}\right) \equiv Z_{M}\left(\tau, Z_{c}, \tau_{t p}, \Theta_{Z}\right)$.

The first reference temperature for the vapor density Eq. (8) is the critical temperature. If the critical quantities $T_{c}, p_{c}, \varrho_{c}$ are kept constant on the same value for all calculations or simulations, there will be no numerical problems.

The critical temperature concerns the model equation ( 7 ) for $Z_{M}\left(\tau, \Theta_{z}\right)$. If during a calculation a simulated critical temperature $T_{\text {sim,c }}$ becomes larger than the critical temperature $T_{c}$ for any reason, then no solution exists or the result is a complex number. The Eqs. (15 to 17 ) must be valid for the critical state,

$f_{\varrho}\left(T_{\text {sim }, c} / T_{c}, \Theta_{p}, \Theta_{z}\right)=1$

$f_{p}\left(T_{\operatorname{sim}, c} / T_{c}, \Theta_{p}\right)=1$

$Z_{M}\left(T_{s i m, c} / T_{c}, \Theta_{z}\right)=Z_{c}$

If these equation constraints are satisfied, there is no need to consider additional constraints on the critical point to the optimization algorithm, see Section 2.1 in reference [3]. The second reference temperature is the triple point temperature, which will be analyzed in more detail in the following section.

\subsection{Investigation of the temperature region around the triple point} temperature

Simulations have shown that the result of parameter estimation leads to solutions that give an excellent fit, but are physically meaningless. Unfortunately, optimization algorithm cannot distinguish between a "mathematical solution" and a "physical solution". As it doesn't exist a lot of experimental vapor density data near the triple point temperature, it increases the difficulty of having a good fitting and so a good model. In order to obtain the good solution with physically meaningful fitted parameters, additional information about the thermodynamic state must be added to the optimization algorithm.

To get a better understanding of the triple point description of the model, the variation of compressibility factor of the vapor phase $Z_{M}\left(\tau, \Theta_{z}\right)$ at the vicinity of the triple point temperature as a function of the reduced temperature has to be analyzed (Fig. 2).

For temperature higher than the triple point temperature there is Vapor Liquid Equilibrium (VLE) and for temperature lower than the triple point temperature there is Vapor Solid Equilibrium (VSE). The lower limit of the model $Z_{M}\left(\tau, \Theta_{Z}\right)$ lies in the VSE region and describes the vapor state as ideal. 
We considered the vapor phase as ideal $\left(Z_{i d}=1\right)$ at an ideal reduced temperature $\left(\tau_{i d}\right)$. This is the point $\left(\tau_{i d}, Z_{i d}=1\right) \cdot \tau_{i d}$ can be calculated considering an adjustable parameter $\widehat{\Theta}_{z 3}$ and the triple point temperature according to Eq. (18)

$\tau_{i d}=\Theta_{z 3} \cdot \tau_{t p}$.

Mathematically, at $\tau_{i d}$ we have the vertex of the model $Z_{M}\left(\tau, \Theta_{z}\right)$ where $Z_{M}\left(\tau_{i d}, \Theta_{z}\right)=1$ and the derivative is equal to zero. Therefore, the product $\Theta_{z 3} \cdot \tau_{t p}$ shall be called reduced ideal gas temperature $\tau_{i d}$ in our model. On the Fig. 2, the point $\left(\tau_{i d}, 1\right)$ with the reduced ideal gas temperature is considered as the weakness of the model. It is difficult to reach ideal gas behavior as pressure must be equal to zero. However, the difference of the real gas behavior from the ideal one will most probably be very small. It is possible in the temperature range $\left.\tau \in] \tau_{i d}, \tau_{t p}\right]$ to extrapolate compressibility factor from the VLE to the VSE regions and to consider that it exists a supercooled vapor below the triple point temperature.

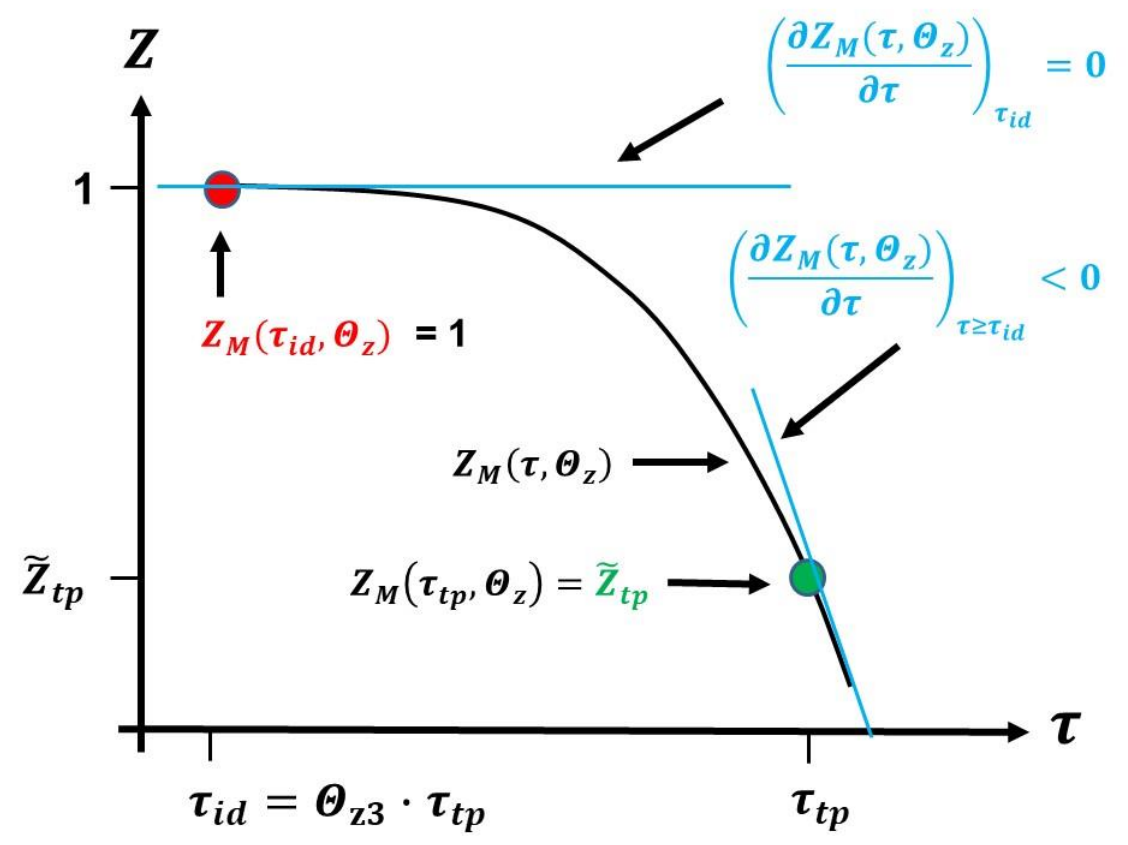

Fig. 2. Variation of the compressibility factor $Z$ for the vapor as a function of the reduced temperature $\tau$ at the vicinity of the reduced triple point temperature $\tau_{t p}$. The objective is to create physically meaningful constraints for the parameterization $\Theta_{z}$ of the $Z_{M}\left(\tau, \Theta_{z}\right)$ model. - Graph of the compressibility factor model $Z_{M}\left(\tau, \Theta_{z}\right)$ for the vapor,

First temperature derivative of the model function $Z_{M}\left(\tau, \Theta_{Z}\right)$ at $\tau_{i d}$ and $\tau_{t p}$. $\tau_{i d}$ "Ideal gas temperature", model specific temperature for which $Z_{M}\left(\tau_{i d}, \Theta_{z}\right)=1$. $\tilde{Z}_{t p} \quad$ Calculated compressibility factor based on reliable data such as measured vapor pressure and vapor density at $T_{t p} \cdot \Theta_{z 3}$ Model parameter which determines $\tau_{i d}$, it must be valid: $\Theta_{z 3}<1$. 
From these considerations, additional reasonable constraints on thermodynamic state variables have to be considered. At the reduced ideal gas temperature $\tau_{i d}$, Eqs. (19) to (21)

$Z_{M}\left(\tau_{i d}, \Theta_{z}\right)=1 \quad\left(\Theta_{z 4, o}=\mathcal{N}_{z}=1\right) \vee\left(\Theta_{z 7, o}=\mathcal{N}_{z}=2\right)$

$\left(\frac{\partial Z_{M}\left(\tau, \Theta_{Z}\right)}{\partial \tau}\right)_{\tau_{i d}}=0$

$\tau_{i d}=\Theta_{z 3} \cdot \tau_{t p}$

At the triple point, Eq. (3) and the derivative of compressibility factor versus reduced temperature Eq. (20) are considered as constraints

$Z_{M}\left(\tau_{t p}, \Theta_{z}\right)-\tilde{Z}_{t p}=0$

$\left(\frac{\partial Z_{M}\left(\tau, \Theta_{Z}\right)}{\partial \tau}\right)_{\tau \geq \tau_{t p}}<0$.

$\tilde{Z}_{t p}$ is the calculated compressibility factor based on trustworthy data. Accurate experimental data or predictions with very well parameterized equations of state like Fundamental Equation of State (FEoS) can be used. With these constraints, we can usefully complete the parameter estimation problem for the vapor density model. But before that, the parameter estimation problem for the evaluation of the vapor pressure data should have to be formulated. In effect, vapor pressure model parameters are needed in the vapor density Eqs. (8) and (9).

2.2 Parameterization of the semi-empirical vapor density model

2.2.1 Formulation of the parameter estimation problem (PE 1) for the evaluation of vapor pressure data

The formulation problem is given by (PE 1). 


$$
\begin{aligned}
& \begin{array}{l}
\text { (PE 1) Formulation of the constrained parameter estimation } \\
\text { problem for vapor pressure evaluation }
\end{array} \\
& \begin{array}{l}
\min _{\Theta_{p}, F_{p}}\left(\bar{Y}_{p}-F_{p}\left(\tau, \Theta_{p}\right)\right)^{\mathcal{T}} \Omega_{p}^{-1}\left(\bar{Y}_{p}-F_{p}\left(\tau, \Theta_{p}\right)\right) \\
\text { s.t. } \quad \Theta_{p 4}=1 \vee 2 \ldots \vee 6 \\
F_{p}\left(\tau_{t p}, \Theta_{p}\right)-p_{t p}=0 \text { optional }
\end{array} \\
& \begin{array}{l}
\bar{Y}_{p} \begin{array}{l}
\text { is the measurement vector of } \\
\text { means with the dimension } \mathcal{N}_{p}
\end{array} \\
\Omega_{p}=\operatorname{diag}\left(\frac{s_{p 1}^{2}}{n_{p 1}}, \frac{s_{p 2}^{2}}{n_{p 2}} \ldots \frac{s_{p N_{p}}^{2}}{n_{p N_{p}}}\right) \quad \begin{array}{l}
\text { is the diagonal matrix of the cor- } \\
\text { responding variances and sample } \\
\text { sizes }
\end{array}
\end{array}
\end{aligned}
$$

$\bar{Y}_{p}$ denotes the experimental data vector with the random variables $\bar{Y}_{p i}, i=$ $1,2 \ldots \mathcal{N}_{p}$. Each $\bar{Y}_{p i}$ is the average calculated value considering the experimental data at the temperature $T_{i}$. The $\bar{Y}_{p i}$ are normally distributed $\bar{Y}_{p i} \sim \mathcal{N}\left(\mu_{p i}, \frac{\sigma_{p i}^{2}}{n_{p i}}\right)$ with the sample sizes $n_{p i}$, the unknown true pressures $\mu_{p i}$ and the corresponding unknown true variances $\frac{\sigma_{p i}^{2}}{n_{p i}}$. $\Omega_{p}$ denotes the variance matrix with the variances of the $\bar{Y}_{p i}$ which are Chi-squared distributed $\frac{\left(n_{p i}-1\right) s_{p i}^{2}}{n_{p i} \sigma_{p i}^{2}} \sim \chi_{\left(n_{p i}-1\right)}^{2} \cdot p_{t p}$ denotes the pressure at the triple point based on measurements or reliable calculations. The result of the optimization considering the formulation problem (PE 1) is the estimated parameter vector $\hat{\Theta}_{p}$. The objective function for parameter estimation is based on the underlying stochasticity. More information can be found in the textbooks by Larsen, Marx [8] and Seber, Wild [9]. If there are enough experimental data near the triple point temperature, the optional constraint of the state variable for the triple point pressure $p_{t p}$ in (PE 1) can be omitted. In such a case, the triple point pressure can be calculated with the estimated parameters according to the Eq. (24)

$\hat{p}_{t p}=F_{p}\left(\tau_{t p}, \hat{\Theta}_{p}\right)$.

If the random standard deviations $S_{i}$ is unknown, then Monte Carlo Simulations (MCS) can be used to simulate a random standard deviations $\mathbb{S}_{p i}$ for the $\Omega_{p}$ matrix

$\Omega_{p}=\bar{n}^{-1} \cdot \operatorname{diag}\left(\mathbb{S}_{p 1}^{2}, \mathbb{S}_{p 2}^{2} \ldots \mathbb{S}_{p \mathcal{N}_{p}}^{2}\right)$,

$\bar{n}$ is a calculated mean sample size for all measurement points. This procedure is described in detail in the supporting material of reference [3]. The 
triple point pressure belongs to the second reference state for the vapor density model and is needed for the calculation of the compressibility factor in the next section.

2.2.2 Formulation of the parameter estimation problem (PE 2) for the evaluation of the vapor density data

Taking into account compressibility factor at ideal reduce temperature (see section 2.1), the parameter estimation problem for the triple point can be described more precisely. According to equation (22), this requires the compressibility factor $\tilde{Z}_{t p}$, which can be calculated for the vapor state at the triple point temperature using vapor pressure $p_{t p}$ and vapor density $\varrho_{t p}$ measurements according to Eq. (26)

$\tilde{Z}_{t p}=\frac{\bar{Y}_{p, t p} \mathcal{M}}{\bar{Y}_{Q, t p} \mathcal{R} T_{t p}}$.

If $\bar{Y}_{p, t p}$ and $\bar{Y}_{Q, t p}$ are not available, the experimental data can be replaced by trustworthy calculations $\tilde{p}_{t p}, \tilde{\varrho}_{t p}$ based on very well parameterized FEoS, so that $\tilde{Z}_{t p}$ can alternatively be calculated according to Eq. (27)

$\tilde{Z}_{t p}=\frac{\tilde{p}_{t p} \mathcal{M}}{\widetilde{\varrho}_{t p} \mathcal{R} T_{t p}}$.

Modeling experience with two terms in the vapor density model for $Z_{M}$ shows that the constraints on the model parameters $\Theta_{z}$ can be actualized to avoid so-called "non-physical" solutions in solving the optimization problem (PE 2). 


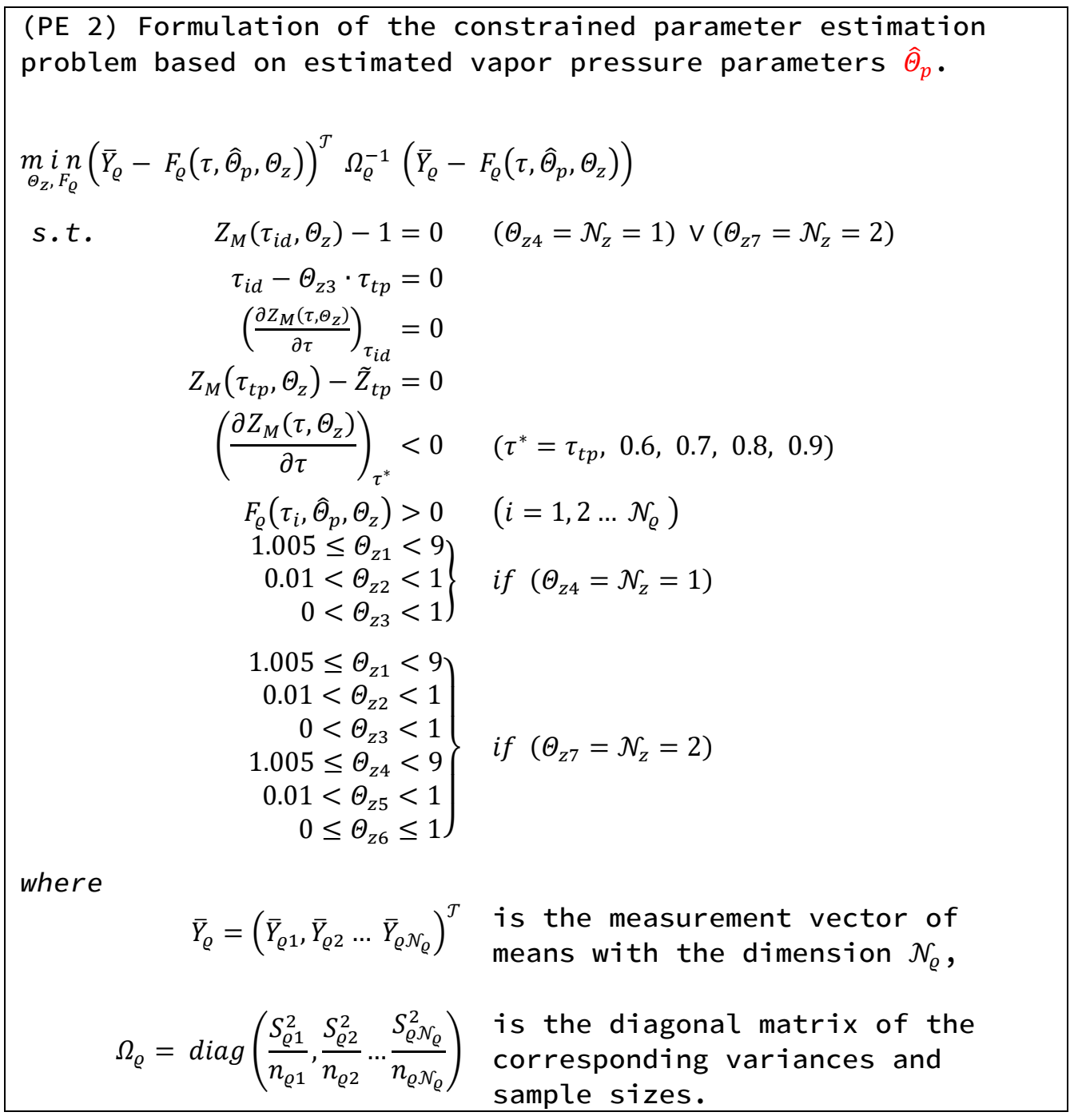

If the standard deviations of the vapor density experimental data are not available, the same procedure as already described for the standard deviations of the vapor pressure measurements can be used (section 2.2.1).

In the (PE2) problem formulation, each equation / inequality with a constraint on the estimation parameters lead to a reduction of the degree of freedom (dof) calculated from all measurement points, if the constraint is reached numerically. The reduction is one dof for each parameter to be estimated. Thus, in this case, the maximum dof reduction can be 3 or 6 units for the inequalities. The dof reduction for the four equations to the state variable constraints is additional four units. In the case of an error propagation calculation for $Z_{M}$, it must be taken into account that the critical quantities also have a measurement error; they must not be assumed to be constant.

If constraints are reached by the parameter estimation algorithm, then the objective function is often not in minimum. This means that an error propa- 
gation calculation must not be calculated according to the equations often quoted which are valid for the minimum.

2.2.3 Formulation of the parameter estimation problem (PE 3) for the evaluation of the vapor density and vapor pressure data simultaneously

As already presented in the previous section, the result of the vapor density parameterization depends on the vapor pressure parameters. As a consequence, the vapor pressure $\hat{p}_{t p}$ estimated from Eq. (24) and the vapor density $\varrho_{M}$ calculated from Eq.(8) may result in a compressibility factor that does not match with the compressibility factor $\tilde{Z}_{t p}$ (Eq.(26) or (27)) determined using experimental values. Moreover, two different compressibility factors exist for the same thermodynamic state at the triple point and it can signify that there is some possible thermodynamic inconsistency. The main reason is attributed to the vapor pressure model, which doesn't take into account the compressibility factor value at the triple point. To avoid this inconsistency, it is recommended to estimate the vapor pressure parameters $\Theta_{p}$ and the parameters $\Theta_{Z}$ simultaneously with the model equation $f_{\varrho}\left(\tau, \Theta_{p}, \Theta_{z}\right)$ (Eqs. (13) and (9)). This means to adjust simultaneously the model's parameters with both vapor pressure and vapor density data. For this purpose, it is necessary to define new vectors of state variables and matrix (Eqs. (28) to $(33))$.

$\bar{Y}:=\left(\begin{array}{c}\bar{Y}_{\varrho} \\ \bar{Y}_{p}\end{array}\right)$

$\Omega_{\varrho}=\operatorname{diag}\left(\frac{s_{\varrho 1}^{2}}{n_{\varrho 1}}, \frac{s_{\varrho 2}^{2}}{n_{\varrho 2}} \ldots \frac{s_{\varrho N_{\varrho}}^{2}}{n_{\varrho N_{\varrho}}}\right)$

$\Omega_{p}=\operatorname{diag}\left(\frac{s_{p 1}^{2}}{n_{p 1}}, \frac{s_{p 2}^{2}}{n_{p 2}} \ldots \frac{S_{p N_{p}}^{2}}{n_{p N_{p}}}\right)$

$\Omega:=\left(\begin{array}{cc}\Omega_{\varrho} & 0 \\ 0 & \Omega_{p}\end{array}\right)$

$\Theta:=\left(\begin{array}{c}\Theta_{z} \\ \Theta_{p}\end{array}\right)$

$F(\tau, \Theta):=\left(\begin{array}{l}F_{\varrho}(\tau, \Theta) \\ F_{p}(\tau, \Theta)\end{array}\right)$

Since these above definitions are very important for the success of the parameterization, the most important equations for the case of two terms in the $Z_{M}$ model (Eq. (7)) shall be formulated explicitly. The parameter vector is defined by Eq. (34). 
$\Theta=\left(\begin{array}{c}\Theta_{1} \\ \Theta_{2} \\ \Theta_{3} \\ \Theta_{4} \\ \Theta_{5} \\ \Theta_{6} \\ \Theta_{7} \\ \Theta_{8} \\ \Theta_{9} \\ \Theta_{10} \\ \Theta_{11}\end{array}\right) \equiv\left(\begin{array}{c}\Theta_{Z 1} \\ \Theta_{Z 2} \\ \Theta_{Z 3} \\ \Theta_{Z 4} \\ \Theta_{Z 5} \\ \Theta_{Z 6} \\ \Theta_{p 1} \\ \Theta_{p 2} \\ \Theta_{p 3} \\ \Theta_{p 4} \\ \mathcal{N}_{z}\end{array}\right)$

The vapor pressure equation is defined by Eq. (35)

$f_{p}(\tau, \Theta)=\exp \left[\frac{\Theta_{7}}{T_{c}}\left(1-\frac{1}{\tau}\right)+\Theta_{8} \cdot \ln (\tau)+\Theta_{9} \cdot T_{c}^{\Theta_{10}}\left(\tau^{\Theta_{10}}-1\right)\right]$.

The reduced vapor density for calculating $F_{Q}(\tau, \Theta)$ in Eq. (33) is given by Eq. (36)

$f_{\varrho}(\tau, \Theta)=Z_{c} \frac{f_{p}(\tau, \Theta)}{\tau \cdot Z_{M}(\tau, \Theta)}$

In Eq. (34), only parameters numbered 1 to 9 are estimated. The number of terms in the $Z_{M}$ model and the integer exponent of the DIPPR 101 equation can be taken from the separate parameterizations from formulations problems $(P E$ 1) and (PE 2). The simultaneous parameter estimation realized in formulation problem (PE 3) will not change these values. 
(PE 3) Formulation of the constrained parameter estimation problem for evaluation of vapor density and vapor pressure data simultaneously

$$
\begin{aligned}
& \min _{\Theta, F}(\bar{Y}-F(\tau, \Theta))^{\mathcal{T}} \Omega^{-\mathbf{1}}(\bar{Y}-F(\tau, \Theta)) \\
& \text { s.t. } \quad Z_{M}\left(\tau_{i d}, \Theta\right)-1=0 \quad\left(\Theta_{8}=\mathcal{N}_{z}=1\right) \vee\left(\Theta_{11}=\mathcal{N}_{z}=2\right) \\
& \tau_{i d}-\Theta_{3} \cdot \tau_{t p}=0 \\
& \left(\frac{\partial Z_{M}(\tau, \theta)}{\partial \tau}\right)_{\tau_{i d}}=0 \\
& Z_{M}\left(\tau_{t p}, \Theta\right)-\tilde{Z}_{t p}=0 \\
& F\left(\tau_{t p}, \Theta\right)_{1}-\varrho_{t p}=0 \\
& \left.F\left(\tau_{t p}, \Theta\right)_{2}-p_{t p}=0\right\} \text { optional } \\
& \left(\frac{\partial Z_{M}(\tau, \Theta)}{\partial \tau}\right)_{\tau^{*}}<0 \quad\left(\tau^{*}=\tau_{t p}, 0.6,0.7,0.8,0.9\right) \\
& F\left(\tau_{i}, \Theta\right)_{1}>0 \quad\left(i=1,2 \ldots \mathcal{N}_{\varrho}\right) \\
& 1.005 \leq \Theta_{1}<9 \text { ) } \\
& \left.\begin{array}{r}
0.01<\Theta_{2}<1 \\
0<\Theta_{3}<1
\end{array}\right\} \text { if }\left(\Theta_{8}=\mathcal{N}_{Z}=1\right) \\
& 1.005 \leq \Theta_{1}<9 \\
& 0.01<\Theta_{2}<1 \\
& 0<\Theta_{3}<1 \\
& 1.005 \leq \Theta_{4}<9 \\
& 0.01<\Theta_{5}<1 \\
& 0 \leq \Theta_{6} \leq 1 \\
& \text { if }\left(\Theta_{11}=\mathcal{N}_{z}=2\right) \\
& \Theta_{7}=1 \vee 2 \ldots \vee 6 \quad \text { if }\left(\Theta_{8}=\mathcal{N}_{z}=1\right) \\
& \Theta_{10}=1 \vee 2 \ldots \vee 6 \quad \text { if }\left(\Theta_{11}=\mathcal{N}_{z}=2\right)
\end{aligned}
$$

where

$$
\begin{gathered}
\bar{Y}=\left(\bar{Y}_{1}, \bar{Y}_{2} \ldots \bar{Y}_{\mathcal{N}_{\varrho}+\mathcal{N}_{p}}\right)^{\mathcal{T}} \begin{array}{l}
\text { is the measurement vector of means } \\
\text { with the dimension } \mathcal{N}_{\varrho}+\mathcal{N}_{p},
\end{array} \\
\Omega:=\left(\begin{array}{cc}
\Omega_{\varrho} & 0 \\
0 & \Omega_{p}
\end{array}\right) \begin{array}{l}
\text { is the diagonal matrix of the cor- } \\
\text { responding variances and sample } \\
\text { sizes. }
\end{array}
\end{gathered}
$$

If the experimental data for the vapor density $\varrho_{t p}$ or the vapor pressure $p_{t p}$ at the triple point are the same regarding their confidence, then neither of the two additional equations for $F_{Q}$ and $F_{p}$ should be used as a state variable constraint in formulation problem (PE 3 ). Both quantities are then consistently estimated by the optimization program.

The simultaneously estimated parameter (formulation problem (PE3)) values can differ significantly from the separately estimated parameters (formula- 
tion problems (PE1) and (PE2)). This is especially true for the $\Theta_{Z}$ parameters. The standard deviations for these parameters can be considerably reduced by considering simultaneous parameterization. The reduction of the standard deviation values of the parameters depends on quality of the database. Taking into account the constraints in the adjustment of the model parameters depends on the trustworthiness of the experimental data including density at the triple point and the vicinity of the triple point.

2.2.4 Capability to predict vapor pressure data from vapor density data based on (PE 4)

If one looks at the vapor density model according to equations (8) and (9), then it should be possible in principle to estimate vapor pressure parameters, even if no vapor pressure data exist. Why? The semi-empirical vapor density model includes the vapor pressure model. In a virtual or mind experiment, we take one experimental vapor pressure data at a time from the database. This increases the standard deviation of the vapor pressure model parameters and also the $Z$ model parameters. The largest standard deviation is achieved in which all experimental vapor pressures data are not considered in the data treatment. The standard deviation of the estimated vapor pressure model parameters depends only on the uncertainty of the experimental vapor density data. Such a parameterization actually works for this model and can be demonstrated via an analysis of the Fisher information matrix [11] or the variance-covariance matrix of the parameters. The interested reader can refer to SuppMat $F$ for more details. Simulations for fluoromethane (R41) and difluoromethane (R32) have shown that the experimental vapor density data have too much uncertainties to obtain satisfactory predictive capability of our model without vapor pressure information. At least one experimental vapor pressure data is recommended with existing experimental vapor density data. Often, the boiling temperature at normal pressure is specified. This vapor pressure information should be provided. The parameter estimation problem (PE4) for this case is formulated as follows. 
(PE 4) Formulation of the constraint parameter estimation problem for the prediction of vapor pressure data from vapor density data with one vapor pressure as a constraint $\min _{\Theta, F}\left(\bar{Y}_{\varrho}-F(\tau, \Theta)\right)^{\mathcal{T}} \Omega_{\varrho}^{-1}\left(\bar{Y}_{\varrho}-F(\tau, \Theta)\right)$

s.t.

$$
\begin{aligned}
Z_{M}\left(\tau_{i d}, \Theta\right)-1 & =0 \quad\left(\Theta_{8}=\mathcal{N}_{z}=1\right) \vee\left(\Theta_{11}=\mathcal{N}_{z}=2\right) \\
\tau_{i d}-\Theta_{3} \cdot \tau_{t p} & =0 \\
\left(\frac{\partial Z_{M}(\tau, \Theta)}{\partial \tau}\right)_{\tau_{i d}} & =0
\end{aligned}
$$$$
Z_{M}\left(\tau_{t p}, \Theta\right)-\tilde{Z}_{t p}=0
$$$$
F\left(\tau_{t p}, \Theta\right)_{1}-\varrho_{t p}=0 \quad \text { Optional }
$$$$
F\left(\tau_{b p}, \Theta\right)_{2}-p_{b p}=0
$$$$
\left(\frac{\partial Z_{M}(\tau, \Theta)}{\partial \tau}\right)_{\tau^{*}}<0 \quad\left(\tau^{*}=\tau_{t p}, 0.6,0.7,0.8,0.9\right)
$$$$
F\left(\tau_{i}, \Theta\right)_{1}>0 \quad\left(i=1,2 \ldots \mathcal{N}_{\varrho}\right)
$$$$
1.005 \leq \Theta_{1}<9
$$$$
\left.0.01<\Theta_{2}<1\right\}
$$$$
\text { if }\left(\Theta_{8}=\mathcal{N}_{z}=1\right)
$$$$
0<\Theta_{3}<1
$$$$
1.005 \leq \Theta_{1}<9
$$$$
0.01<\Theta_{2}<1
$$$$
0<\Theta_{3}<1
$$$$
1.005 \leq \Theta_{4}<9
$$$$
\text { if }\left(\Theta_{11}=\mathcal{N}_{z}=2\right)
$$$$
0.01<\Theta_{5}<1
$$$$
0 \leq \Theta_{6} \leq 1
$$

$$
\begin{aligned}
\Theta_{7} & =1 \vee 2 \ldots \vee 6 & & \text { if }\left(\Theta_{8}=\mathcal{N}_{z}=1\right) \\
\Theta_{10} & =1 \vee 2 \ldots \vee 6 & & \text { if }\left(\Theta_{11}=\mathcal{N}_{z}=2\right)
\end{aligned}
$$

where

$$
\begin{gathered}
\bar{Y}_{\varrho}=\left(\bar{Y}_{\varrho 1}, \bar{Y}_{\varrho 2} \ldots \bar{Y}_{\varrho \mathcal{N}_{\varrho}}\right)^{\mathcal{T}} \begin{array}{l}
\text { is the measurement vector of } \\
\text { means with the dimension } \mathcal{N}_{\varrho},
\end{array} \\
\Omega_{\varrho}=\operatorname{diag}\left(\frac{S_{\varrho 1}^{2}}{n_{\varrho 1}}, \frac{S_{\varrho 2}^{2}}{n_{\varrho 2}} \ldots \frac{S_{\varrho \mathcal{N}_{\varrho}}^{2}}{n_{\varrho \mathcal{N}_{\varrho}}}\right) \begin{array}{l}
\text { is the diagonal matrix of the } \\
\text { corresponding variances and } \\
\text { sample sizes. }
\end{array}
\end{gathered}
$$

\subsection{Assessment quantities}

The experimental data are necessary for the parameterization of our model. The data are analyzed, checked for outliers and not take into account if necessary. The results of the analysis of the data base used in this work is presented in Appendix B. The databases for the evaluation are provided and are listed in "Data References" from [dataset 1] to [dataset 4]. The 
definition of the objective function results in the parameter values, with which the following evaluation variables are calculated:

a) Mean of Relative Deviation in \% $(M R D \%(U))$

b) Maximum of Mean of Relative Deviation in \% $(\max R D \%)$

c) Model Bias $(\operatorname{Bias} \%(U))$

d) Rank ( $R k$ ) of variance-covariance matrix (cov) of the estimated parameter

$U$ denotes the vapor density or vapor pressure. These indicators are calculated in general way according to the Eqs. (37) to (39)

$M R D \%(U, \widehat{\Theta})=\frac{100 \%}{\mathcal{N}_{U}} \sum_{i=1}^{\mathcal{N}_{U}}\left|\frac{\bar{Y}_{U, i}-F_{U}\left(\tau_{i}, \widehat{\Theta}\right)}{\bar{Y}_{U, i}}\right|$

$\max M R D \%(U, \hat{\Theta})=100 \% \cdot \max \left[\left|\frac{\bar{Y}_{U, i}-F_{U}\left(\tau_{i}, \widehat{\Theta}\right)}{\bar{Y}_{U, i}}\right| \quad i=1,2 \ldots \mathcal{N}_{U}\right]$

$\operatorname{Bias} \%(U, \widehat{\Theta})=\frac{100 \%}{\mathcal{N}_{U}} \sum_{i=1}^{\mathcal{N}_{U}}\left(\frac{\bar{Y}_{U, i}-F_{U}\left(\tau_{i}, \widehat{\Theta}\right)}{\bar{Y}_{U, i}}\right)$.

The rank (Rk) provides information whether the parameters can be estimated independently or not. Also it permits to check if there is enough experimental data to estimate all the parameters or only some of them.

In the context of evaluating the vapor pressure prediction capability, two general assessment quantities have to be defined. The first one concerns the general assessment of a regression in which the estimated state variable (e.g., vapor pressure) is obtained from a corresponding experimental data (vapor pressure experimental data). It is not only used for testing the predictive capability, but can also be used in general. The second one concerns a special assessment where the estimated state variable (e.g. vapor pressure) is obtained from another experimental data (e.g. vapor density measurement). For both quantities, it is a quotient of the number of experimental data $\mathcal{N}_{Y, \text { conf }}$ described by the model in a confidence interval of the data value and the total number of measurements $\mathcal{N}_{Y}$ of the random variable $Y$ (e.g. vapor pressure). If there are corresponding variables, $\mathcal{N}_{Y, \text { conf }}$ depends on the database $Y$ and is referred to $\mathcal{N}_{Y, \text { conf }}(Y)$. If the number depends on the non-corresponding database $X$, it is referred to $\mathcal{N}_{Y, \text { conf }}(X)$. In this case, the regression runs on $X$. Thus, the so-called "fit capability" and the "predictive capability" can be defined by Equations (40) and (41).

FitCap $\% \equiv$ FitCap $\%_{Y Y}:=\frac{\mathcal{N}_{Y, \text { conf }}(Y)}{\mathcal{N}_{Y}} \cdot 100 \%$

$\operatorname{PreCap} \% \equiv \operatorname{PreCap} \%_{X Y}:=\frac{\mathcal{N}_{Y, \text { conf }}(X)}{\mathcal{N}_{Y}} \cdot 100 \%$

The number $\mathcal{N}_{Y, \text { conf }}$ is also identical to the number of residuals whose absolute value is smaller than a given accepted deviation. Thus, this assessment quantity can be easily calculated from the residuals. PreCap\% can most likely be applied to the assessment of EoS as well. These assessment quantities are rigorous if the measurement data base is free of outliers and the accepted deviation between model and measurement is realistically chosen. The meaningfulness of these two quantities is lost by "screwing" with the ac- 
cepted deviation, because $100 \%$ can be reached easily. Therefore, the accepted deviation, which is incidentally related to the confidence interval conf, should also be specified for this variable.

In the case of the outlier test for the experimental vapor pressure data, a CoV\% value of $0.5 \%$ was assumed as the maximum relative deviation acceptable. This value was also used for the calculation of FitCap $\%$ and PreCap\% indicators.

Using MCS, mean values and standard deviations of the parameters and the above-mentioned assessment variablescan be calculated. The estimated model parameters and the assessment quantities become more robust by using MCS, because the fluctuations of the standard deviations are taken into account. In this context, average values of thermodynamic properties, such as boiling temperature, triple point pressure or vapor density at the triple point, can also be determined using MCS. All the details concerning the utilization Monte Carlo simulations are described in Appendix C. All calculations were made with the software Mathcad 15 [12].

\section{Results and Discussions}

Statistical analysis of the raw data estimation of standard deviations and analysis of the outliers are some results of the parametrization. The results are presented in Supplementary Material (SuppMat) B. Also, the analysis of the properties of the pure compounds are presented in SuppMat $A$.

First, comparison between the results obtained by separate and simultaneous evaluation are discussed in terms of model's parameter values, then the model evaluation, the calculated state variables, and finally the state of the art. We would like to focus the discussion of individual results on only one compound (fluoromethane (R41)) in order to describe the principal method and thus streamline the content. The detailed results concerning difluoromethane (R32) are presented in a Supplementary Material.

The separate evaluations for vapor pressure and vapor density were calculated according to the formulation problems (PE 1) and (PE 2). This means that the estimated vapor density parameters depend on the previously, separately estimated vapor pressure parameters. The optional constraint in the formulation problem (PE1) for vapor pressure at the triple point temperature was not enabled. The simultaneous parameter estimation was calculated according to formulation problem (PE 3) without the optionally specified restrictions at the triple point, so that degrees of freedom exist to achieve thermodynamic consistency.

The estimated model's parameters for fluoromethane (R41) are presented in Table 1. Considering the three formulation problems, we can observe that the parameters for the vapor pressure model change only slightly. The two parameter vectors are also identical in a statistical sense. Only, the 
parameters $\bar{\Theta}_{Z, 1}$ have significant different values. The results of simultaneous evaluation also show a significantly larger number value for the parameter $\bar{\Theta}_{Z, 6}$, which is important for the description of compressibility factor close to critical point. The parameter $\bar{\Theta}_{Z, 3}$ estimates the modelspecific, so-called ideal gas temperature $T_{i d}$. The smaller $\bar{\Theta}_{Z, 3}$ is, the smaller $T_{i d}$ is in comparison to the triple point temperature and the greater is the assurance to be able to extrapolate to temperature lower than the triple point temperature where we have a supersaturated liquid. It should be mentioned that the result of the parameter estimation does not assign the first term to the critical temperature range in all cases. As is well known, the result of a nonlinear parameter estimation depends very much on the starting values, which are often element of a fractal.

Table 1

Estimated parameters based on the formulation of the parameter estimation problems (PE 1) to (PE 3) for fluoromethane (R41). Results are based on Monte Carlo Simulations.

\begin{tabular}{|c|c|c|c|c|c|c|c|}
\hline \multirow{2}{*}{$\begin{array}{l}\text { Estimated } \\
\text { Parameter }\end{array}$} & \multicolumn{2}{|c|}{$\mathrm{PE} 1^{1)}$} & \multicolumn{2}{|c|}{$\mathrm{PE} 2^{2)}$} & \multicolumn{2}{|c|}{ PE $3^{3)}$} & \multirow{2}{*}{$\begin{array}{c}\text { Remarks on the Responsibility } \\
\text { and Meaning of Parameters }\end{array}$} \\
\hline & Average & Cov\% & Average & CoV\% & Average & CoV\% & \\
\hline $\bar{\theta}_{p 1}$ & 3022.3 & 0.039 & & & 3023.8 & 0.038 & \\
\hline $\bar{\Theta}_{p 2}$ & -5.967 & 0.142 & & & -5.977 & 0.138 & exponent $\theta_{p 4, o p t}=2$ \\
\hline $\bar{\theta}_{p 3} \cdot 10^{5}$ & 1.5648 & 0.181 & & & 1.5675 & 0.177 & \\
\hline $\bar{\theta}_{z 1}$ & & & 8.772 & 7.6 & 1.626 & 5.3 & mainly for the $T_{c}$ region \\
\hline $\bar{\theta}_{z 2}$ & & & 0.230 & 4.2 & 0.235 & 3.6 & mainly for the $T_{C}$ region \\
\hline $\bar{\theta}_{z 3}$ & & & 0.706 & 3.5 & 0.945 & 1.0 & for ideal gas temperature ${ }^{4)}$ \\
\hline $\bar{\Theta}_{z 4}$ & & & 4.160 & 3.4 & 4.249 & 3.7 & for the $T_{c}$ and $T_{t p}$ region \\
\hline $\bar{\Theta}_{z 5}$ & & & 1.561 & 4.8 & 1.824 & 6.9 & for the $T_{c}$ and $T_{\text {tp }}$ region \\
\hline $\bar{\theta}_{z 6}$ & & & 0.380 & 4.3 & 0.555 & 3.6 & fraction of $T_{c}$ region description \\
\hline
\end{tabular}

1) vapor pressure data only. 2) vapor density data only. 3) vapor pressure and vapor density data simultaneously.

For simulations much more digits are necessary, see appendix 4. 4) model specific temperature.

Guess parameter values in the convergence region are suggested (see Appendix D). The same applies to the parameters of the vapor pressure equation. In the context of the assignment of the two terms in the $Z$ function (eq (7)) to a temperature range, an analysis of the graphs can provide information. Fig. 3 shows the first term $\Theta_{z 6}\left[1-\left(\frac{\tau-\theta_{z 3} \cdot \tau_{t p}}{1-\theta_{z 3} \cdot \tau_{p}}\right)^{\theta_{z 1}}\right]^{\theta_{z 2}}$ and second term $\left(1-\Theta_{z 6}\right)\left[1-\left(\frac{\tau-\theta_{z 3} \cdot \tau_{t p}}{1-\theta_{z 3} \cdot \tau_{t p}}\right)^{\theta_{z 4}}\right]^{\theta_{z 5}}$ with the components $\bar{\Theta}_{z 6}$ and $\left(1-\bar{\Theta}_{z 6}\right)$, respectively. It can be clearly seen that the first term is predominantly responsible for describing compressibility factor $Z$ in the temperature range below the critical temperature. The low and medium temperature range is described by both 
terms. Table E.1 in Appendix E presents the model's parameters obtained for difluoromethane (R32).

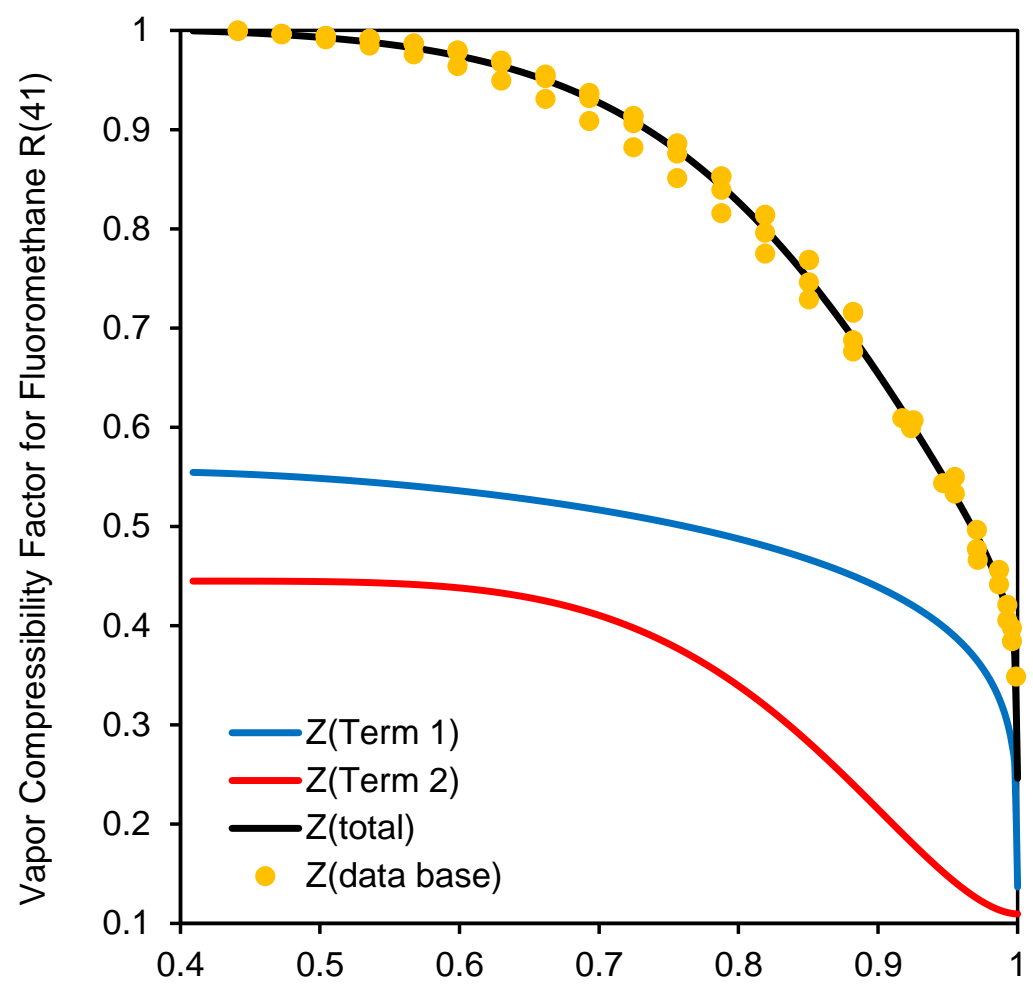

Reduced Temperature

Fig. 3. Compressibility factor for fluoromethane (R41) vapor vs. reduced temperature. The total compressibility factor is the sum of the first and second term according to eq (7). Term 1 is mainly responsible for the description of the critical temperature range. The fraction for the $1^{\text {st }}$ term is $\bar{\Theta}_{z 6}=0.555$ and that for the $2^{\text {nd }}$ term is $\left(1-\bar{\Theta}_{z 6}\right)$. Compressibility factors $(0)$ calculated from vapor densities and the vapor pressure equation (5) with the parameters from (PE 3) with the vapor densities from the database. $Z_{c}=0.2462$.

Table 2 shows the results for the different evaluations concerning fluoromethane (R41). The MRD\% values for the vapor pressure are equally small for both evaluations of the formulation problems (PE 1) and (PE 2). Also, it shows a very good agreement between model assumption and experimental data. The maximum RD\% value is acceptable, as it still belongs to the range of variation of $0.5 \%$.

The optimal value for the model bias $(\overline{B l a s \%})$ is a statistical zero for experimental data. Both evaluations show a zero value because the standard deviation is very large in comparison to the average value. In this case, however, it is not a statistical zero because the residuals are not randomly distributed around zero. The residuals $\overline{R D \%}(p)_{i}$ values in Fig. E.5 (SuppMat 
E) show clear structures. This is due to the correlated database. A very similar residual structure can also be seen in the publication by Lemmon et al. [14].

Table 2

Regression assessment for the different kind of parameter estimation formulations (PE 1) to (PE 3) for fluoromethane (R41). The variance-covariance matrix of the parameters has full rank in any case.

\begin{tabular}{lcccccc}
\hline & \multicolumn{2}{c}{ PE 11) } & \multicolumn{2}{c}{ PE 2) } & \multicolumn{2}{c}{ PE 33) } \\
Assessment Quantity & Average & Stdev & Average & Stdev & Average & Stdev \\
\hline MRD\% for vapor pressure & 0.166 & 0.0004 & & & 0.166 & 0.0005 \\
maxRD\% for vapor pressure & 0.510 & n.r. & & & 0.516 & n.r. \\
Bias\% for vapor pressure & -0.011 & 0.0036 & & & -0.008 & 0.004 \\
MRD\% for vapor density & & & & & & \\
maxRD\% for vapor density & & & 1.091 & 0.015 & 1.113 & 0.012 \\
Bias\% for vapor density & & & 4.781 & n.r. & 4.734 & n.r. \\
\hline
\end{tabular}

1) vapor pressure data only. 2) vapor density data only. 3) vapor pressure and vapor density data simultaneously. n.r. is not recommended

Concerning the vapor density, the two mean errors (MRD\%) are equal considering a statistical point of view and are also in good agreement with the known uncertainty of the experimental vapor density data. The residual plots (Fig. E.4 and Fig. E.5) for vapor density and vapor pressure are detailed in SuppMat E. The maximum MRD\% value is about five times larger than the MRD\% value for both evaluations. However, the residuals analysis in Fig. E.4 shows that this deviation belongs to a measurement point that is not an outlier. Thus, we can consider that it is not a systematic deviation. The bias is close to zero value for both analyses, since the standard deviation is much larger than the mean itself. This zero value signifies that we have realized a very good and balanced regression. Both evaluations indicate a very good score for the regression. Table E.2, Fig. E.8 and Fig. E.9 in SuppMat $E$ show the results for difluoromethane.

First, the relevant, physical quantities such as triple point pressure, vapor density at the triple point and the associated compressibility factor are discussed and then the quantities for the boiling point.

For the separate evaluation according to formulation problems (PE 1 ) and (PE 2), the predicted data by REFPROP 10.0 software and the results from formulation problems (PE 1) and ( $P E$ 2) are compared. The results from formulation problem (PE 3) may not be included concerning the discussion of 
the separate evaluation. Table 3 presents the results. In this work and for the simulations physical properties (constants), parameters and other quantities are considered. It is well known that in nonlinear modeling smallest deviations of these quantities can cause large deviations in the results. In order to have identical results between the different working groups and the same exactness, it is important to consider numerical values with many digits.

Table 3

Comparison of the results of the physical properties of fluoromethane (R41) for the different parameter estimation formulations (PE 1) to (PE 3). Results are based on Monte Carlo Simu-lations.

\begin{tabular}{|c|c|c|c|c|c|c|c|}
\hline \multirow[b]{2}{*}{ Quantity } & \multirow[b]{2}{*}{ Database ${ }^{(4)}$} & \multicolumn{2}{|l|}{$\mathrm{PE} 1^{(1)}$} & \multicolumn{2}{|l|}{$\mathrm{PE} 2^{(2)}$} & \multicolumn{2}{|c|}{$P E 3^{(3)}$} \\
\hline & & Average & Stdev & Average & Stdev & Average & Stdev \\
\hline Boiling point temperature $T_{b} / K$ & 194.97 & 194.84 & 0.001 & & & 194.84 & 0.001 \\
\hline Boiling point temperature $/{ }^{\circ} \mathrm{C}$ & -78.18 & -78.31 & 0.001 & & & -78.31 & 0.001 \\
\hline Boiling point vapor density $/ \mathrm{kg} / \mathrm{m}^{3}$ & 2.238 & & & 2.190 & 0.002 & 2.195 & 0.001 \\
\hline Triple point vapor pressure / $\mathrm{Pa}$ & 345.04 & 347.0777 & n.r. & $347.0798^{(6)}$ & & 346.9314 & n.r. \\
\hline Triple point vapor density $/ \mathrm{kg} / \mathrm{m}^{3}$ & 0.010884 & & & 0.010948 & n.r. & 0.010944 & n.r. \\
\hline Compressibility factor at triple point & 0.999544 & $1.005447^{(5)}$ & & 0.999550 & & 0.999539 & n.r. \\
\hline "Ideal gas temperature" / K & & & & 91.61 & 3.23 & 122.63 & 1.24 \\
\hline
\end{tabular}

(1) vapor pressure data only. (2) vapor density data only. (3) vapor pressure and vapor density data simultaneously. (4) calc with the FEoS in REFPROP 10.0, except $T_{b}$ which is a mean from measurements.

(5) calculated $Z_{\mathrm{tp}}$ from $p_{\mathrm{tp}}(P E$ 1) and vapor density (database). (6) vapor pressure calculated from compressibility factor and vapor density both from (PE 2). n.r. not recommended.

From the estimated vapor pressure value of $347.0777 \mathrm{~Pa}$ at the triple point according to formulation problem (PE 1) and the vapor density value of $0.010884 \mathrm{~kg} / \mathrm{m}^{3}$ from the data base, we calculate a compressibility factor value of 1.005447 , which is greater than 1 and thus physically nonsensical.

With the formulation problem (PE 2), we calculate a vapor density value of $0.010948 \mathrm{~kg} / \mathrm{m}^{3}$ and a compressibility factor value of 0.999550 , which is less than 1 and thus physically meaningful. From this, a vapor pressure value of $347.0798 \mathrm{~Pa}$ is calculated, which is not identical with the vapor pressure of $347.0777 \mathrm{~Pa}$ obtained with formulation problem (PE 1). Even if one calculates a vapor density from the vapor pressure obtained with formulation problem (PE 1) and the compressibility factor obtained with formulation problem (PE 2), the result is different from the vapor density value obtained with formulation problem (PE 2)! The reason of these differences is probably due to the restriction applied to the compressibility factor in formulation problem (PE 2). During the minimization of the sum of the deviation squares, inevitably also the compressibility factor is slightly optimized to obtain 
an optimal vapor density description. For both evaluations according to formulation problems ( $P E 2$ ) and (PE 3), different compressibility factors are optimized as well.

There are for the separate evaluation

- three different triple point pressure values (345.04, 347.0777, 347.0798) $\mathrm{Pa}$,

- at least two different vapor density values $(\odot .010884,0.010948) \mathrm{kg} / \mathrm{m}^{3}$ and

- three different compressibility factor values ( $0.999544,1.005447$, $\odot .999550)$.

Should one choose the result of formulation problem (PE 2) from this "rich offer", because the vapor density model is clever and involves the vapor pressure equation, Moreover, still the inconsistency concerning different vapor pressure description between formulation problems (PE 1) and (PE 2) remains.

In the case of a simultaneous evaluation according to formulation problem (PE 3), the parameters are estimated in such a way that vapor pressure and vapor density state variables lead to one compressibility factor value thermodynamically consistent. For this reason, the simultaneous evaluation is preferable.

In view of the small differences, one can think that it does not matter whether the separate or simultaneous evaluation is calculated. But it is a question of recognizing the principal differences of the evaluation in the light of the thermodynamic consistency. The differences between the results obtained from formulation problems (PE1) and (PE2) turn out to be so small numerically here because the data basis is very good in the range of the triple point temperature. If the data basis is more uncertain near the triple point temperature, then most likely the differences between the evaluations will also be larger.

Table 3 still lists the physical properties at the fluoromethane (R41)'s boiling point. Considering the evaluations according to formulation problems ( $P E$ 1) and (PE 3), the same boiling temperature of $194.84 \mathrm{~K}$ are calculated. The value is also identical to that calculated by REFPROP 10.0 software. The vapor density at the boiling point is about 2\% lower than that calculated by REFPROP 10.0. Table E.3 in SuppMat $E$ shows the results obtained for difluoromethane (R32).

Often thermodynamic properties in the temperature range below the critical temperature are of great interest. But many equations of state, including fundamental equations, show weaknesses in the prediction of the thermodynamic properties in this temperature range. For comparison, compressibility factor was also calculated using the FEoS implemented in REFPROP 10.0 and 
the very commonly used PR EoS involving the Mathias Copeman (MC) a function [15]. The MC parameters for the PR EoS were fitted to the vapor pressure equation and are listed in Appendix E for Fig. E.4. The model equations were compared with experimental data that exist in this temperature range. Fig. 4 shows the results for fluoromethane (R41).

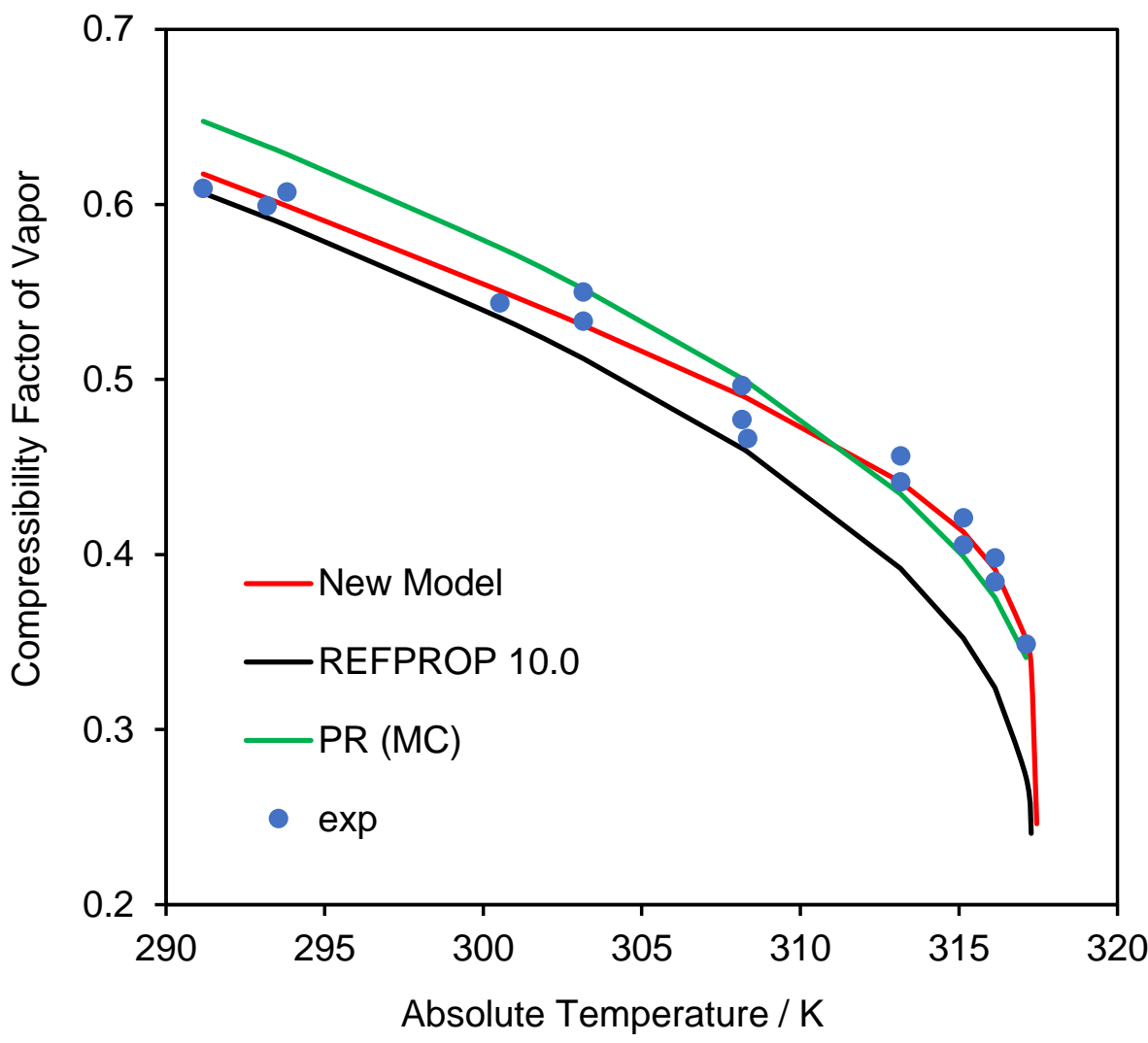

Fig. 4. Comparison of experimental data from fluoromethane (R41) and predicted compressibility factor for vapor from different models vs. absolute temperature near the crititical temperature $\left(T_{c}=317.454 K, Z_{c}=0.24623\right)$. PR (MC) denotes Peng Robinson EoS with Mathias Copeman $\alpha$ function. The uncertainty for the new model $\left(Z_{M}\right)$ is $\sim 0.03$ which is calculated by error propagation. $Z(\exp )$ was calculated from the vapor density measurements and the vapor pressure function with the parameters according to formulation problem (PE 3 ).

In addition to the graphical representation, the statistical assessment quantities, presented in Table 4, were calculated for this temperature range. From Table 4, we can observe that only the semi-empirical vapor density model prediction are in good agreement with experimental data. The FEOS available in REFPROP 10.0 software shows the largest deviations, followed by the PR EoS.

Experimental vapor densities data of difluoromethane can be described very well by our new model. The relative deviations are presented in Fig. E.8 in SuppMat $\mathrm{E}$. 
Table 4

Assessment comparison of the new model with the state of the art in the temperature range below the critical temperature. The basis for the comparison is the com-pressibility factor for fluoromethane (R41) calculated from measured (exp) vapor densities. The values given are average values.

\begin{tabular}{lccc}
\hline Assessment & New Model & $\mathrm{RP}^{1)} 10.0$ & $\mathrm{PR}(\mathrm{MC})^{2)}$ \\
\hline Average MRD\% & 1.79 & 10.6 & 3.81 \\
Average maxRD\% & 4.69 & 29.0 & 6.51 \\
Average Bias\% & 0.086 & -10.6 & 0.74 \\
\hline
\end{tabular}

1) REFPROP. 2) PR EoS with Mathias Copeman a function

Now to the results of the exciting question whether vapor pressure data can be predicted from vapor density data. The following strategy is recommended for this:

1. Take all reliable vapor density data and only one vapor pressure data, e.g. at the boiling point or better activate a contraint for this point. Calculate the parameterization for all parameters based on the problem formulation (PE 4) for all parameters. Result is $\hat{\Theta}$.

2. Extract the parameter part from $\hat{\Theta}$ used for the vapor pressure prediction.

3. Calculate with this part of parameter vector the predicted vapor pressure data only and compare the values with the experimental vapor pressure data.

It is recommended to select a vapor pressure value in the middle temperature range of the vapor pressure curve to provide information about the convexity of the vapor pressure model to the optimization algorithm.

The results for fluromethane and difluoromethane are listed in Table 5. For better comparison, the results from formulation problem (PE 3) are also listed. Fig. E.6 and E.7 in SubbMat E show the corresponding residuals $\mathrm{RD} \%(\mathrm{p})$.

First of all, it can be stated that in principle it is possible to predict the vapor pressure from vapor density using the empirical vapor density model. The condition is that only a trustworthy vapor pressure information in the middle of temperature range should be provided for the present database. This is not possible with the empirical vapor density models known so far like Wagner type equations.

From Table 5 it can be seen that the MRD\% values for the formulation problem (PE 4) evaluation become larger, but are still in a range that is classified as good or satisfactory. The Bias\% values indicate a systematic deviation for both compounds (see Fig. E.6 and E.7), which can also be seen in the residual plots. 
Table 5

Assessment of predictive capability PreCap\% of vapor pressure data based on vapor density data and the evaluation according to formulation problem (PE 4) with one constraint at $\tau_{b p}$. The number of vapor pressure data to be predicted is $\mathcal{N}_{p, R 41}=293$ and $\mathcal{N}_{p, R 32}=716$.

\begin{tabular}{|c|c|c|c|c|}
\hline \multirow[b]{2}{*}{ Assessment Quantity } & \multicolumn{2}{|c|}{ Fluoromethane (R41) } & \multicolumn{2}{|c|}{ Difluoromethane (R32) } \\
\hline & (PE 3) & (PE 4) & (PE 3) & (PE 4) \\
\hline Average MRD\% for vapor pressure & 0.166 & 0.465 & 0.171 & 0.989 \\
\hline Average maxRD\% for vapor pressure & 0.516 & 1.230 & 0.512 & 1.911 \\
\hline Average Bias\% for vapor pressure & -0.008 & 0.387 & -0.017 & -0.755 \\
\hline FitCap\%1) & 99.3 & & 99.9 & \\
\hline PreCap\% ${ }^{11}$ & & 53.2 & & 16.1 \\
\hline Rank of cov matrix $\left.{ }^{2}\right)$ of the parameter & 9 & 9 & 9 & 9 \\
\hline
\end{tabular}

1) maximal accepted deviation: $0.5 \%$. 2) Expected full rank of the variance-covariance matrix: 9

For the assessment according to formulation problem (PE 3 ), the Fit Capability FitCap\% shows that more than 99\% of the experimental vapor pressure data can be described by the model. In contrast, the predictive power PreCap\% predicts 53\% of the vapor pressure data for fluoromethane and only 16\% for difluoromethane. The reason for the much lower predictive capability compared to the fit capability is the quality of the experimental vapor density data. The measurement errors are larger for difluoromethane than for fluoromethane.

The predictive capability PreCap\% can be significantly increased, but this has its price. Either experimental vapor density data with better accuracy and precision are measured in the laboratory, or either two trustworthy experimental vapor pressure data are provided. Qualitatively better experimental vapor density data are not available for the simulations within the scope of this work. Therefore, the predictions are calculated using two experimental vapor pressures data as constraints at two reduced temperatures $\tau_{1}=0.5$ and $\tau_{2}=0.8$, which were set arbitrarily. These two vapor pressures should come from very trustworthy measurements or calculations. In this case, experimental vapor pressure data were selected from the database with very small RD\%(p) values. The results are listed in Table 6.

With only one more experimental vapor pressure data, more than 96\% of the 293 or 716 experimental vapor pressure data can be predicted with a maximum error of $\pm 0.5 \%$.

Now, three vapor pressure values could be formulated as constraints to achieve even greater accuracy. However, this must not be done, because there is no degree of freedom remaining to estimate the third vapor pressure parameter. 
Assessment of predictive capability PreCap\% of vapor pressure data based on vapor density data and two vapor pressure data at $\tau_{1}=0.5$ and $\tau_{2}=0.8$ as constraints formulated in formulation problem (PE 4). The number of vapor pressure data to be predicted is $\mathcal{N}_{p, R 41}=293$ and $\mathcal{N}_{p, R 32}=716$.

\begin{tabular}{lcc}
\hline & $\begin{array}{c}\text { Fluorome- } \\
\text { thane (R41) }\end{array}$ & $\begin{array}{c}\text { Difluorome- } \\
\text { thane (R32) }\end{array}$ \\
\hline Assessment Quantity & 0.193 & 0.178 \\
Average MRD\% for vapor pressure & 0.720 & 0.566 \\
Average maxRD\% for vapor pressure & 0.013 & 0.009 \\
PreCap\% ${ }^{1)}$ & 96.6 & 99.4 \\
Rank of cov matrix ${ }^{2)}$ of the parameter & 9 & 9 \\
\hline
\end{tabular}

1) maximal accepted deviation: $0.5 \%$. 2) Expected full rank: 9

Three unknown vapor pressure parameters are determined by three equations (constraints) and are not estimated. In this case, the number of degrees of freedom is zero. This should not be allowed in the statistical sense. In effect, the number of degrees of freedom should be as large as possible. If more than two vapor pressure data are used, then the evaluation must be done according to the problem formulation (PE 3 ). Using Model-based Design of Experiments, the optimum temperatures of the vapor pressure data can be calculated.

The predictive capability according to the formulation problem (PE 4) can be used to check experimental vapor density data and also SeM for thermodynamic consistency. The following questions can be answered:

- Do the vapor density data match with the experimental vapor pressure data?

- Are the vapor density and vapor pressure data thermodynamically consistent?

- With known thermodynamic consistency of the experimental vapor density and experimental vapor pressure data, is the semi-empirical vapor density model appropriate for the compound under investigation?

\section{Conclusions}

The semi-empirical model can now be used to describe vapor densities for pure compounds for the full range from the triple point to the critical temperature. It was successfully verified using fluoromethane (R41) and difluoromethane (R32). This model can also be used to verify the reliability of vapor density data. The model can predict vapor pressure from vapor density data with only one reliable vapor pressure information. The predictive capability can be highly improved by providing two vapor pressure 
data at two different temperatures. In this case, for fluoromethane and difluoromethane, more than $96 \%$ of the vapor pressure data can then be predicted with a relative error of $\leq 0.5 \%$. The predictive capability of the vapor pressure is strongly dependent on the quality of the vapor density data. For a supercooled liquid, the compressibility factor for the vapor phase could be extrapolated to a near temperature range below the triple point temperature. The uncertainty can be given by error propagation. Its value depends on the quality of the vapor density and vapor pressure data at the vicinity of the triple point temperature. In any case, the predictions need to be verified experimentally which is not in the scope of this work. The compressibility factor for the vapor of fluoromethane and difluoromethane according to the $Z$-model agrees well with the average compressibility factor based on molecular simulations according to Eggimann et al. [5] within the limits of the mean deviations. In principle, molecular simulations according to Eggiman et al. can also be used to obtain additional information on whether the semi-empirical vapor density model is suitable for modeling a compound. We are optimistic that this semi-empirical model with two terms can be applied to other compounds as well. The precondition is that there are no large fractions of oligomers in the vapor phase. 


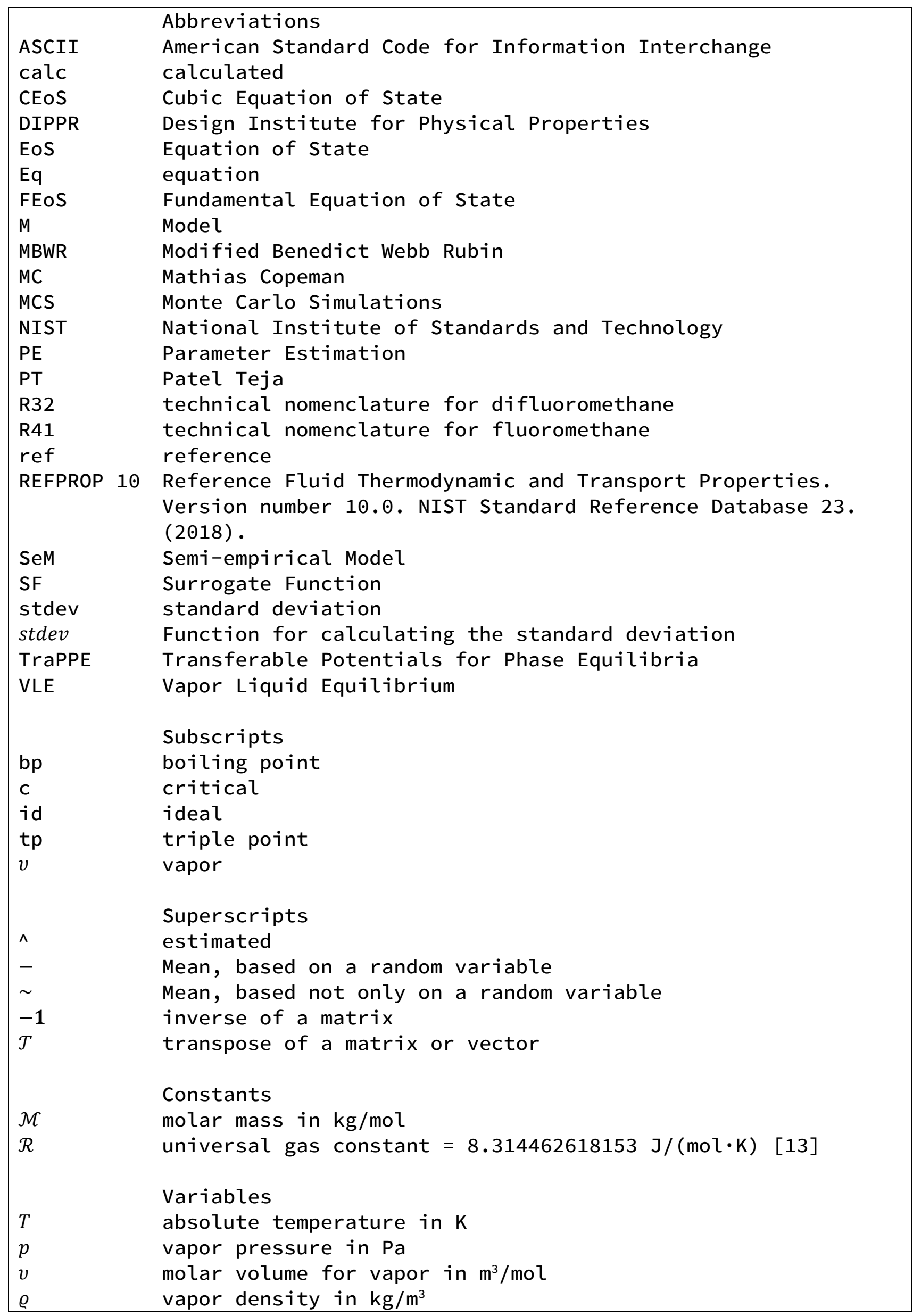




\begin{tabular}{|c|c|}
\hline$\tau$ & reduced temperature, dimensionless \\
\hline$\omega$ & reduced vapor density, dimensionless \\
\hline$\pi$ & reduced vapor pressure, dimensionless \\
\hline$\dot{\omega}$ & acentric factor \\
\hline$Z$ & compressibility factor for vapor, dimensionless \\
\hline$n$ & quantity of matter in mol \\
\hline$U$ & general placeholder for a state variable \\
\hline$\Theta$ & $\begin{array}{l}\text { Statistical variables and general quantities } \\
\text { parameter vector }\end{array}$ \\
\hline$\Omega$ & variance-covariance matrix of the measurements \\
\hline conf & confidence region on a random variable \\
\hline $\operatorname{cov}$ & variance-covariance matrix of the parameters \\
\hline $\mathrm{CoV}$ & Coefficient of Variation \\
\hline $\operatorname{CoV} \%$ & Coefficient of Variation in $\%(\operatorname{CoV} \%=\operatorname{CoV} \cdot 100 \%)$ \\
\hline $\bar{Y}_{i}$ & $\begin{array}{l}\text { mean of measured random variable at temperature } T_{i} \quad(i= \\
1,2 . \mathcal{N}) \text {, in } \mathrm{kg} / \mathrm{m}^{3} \text { or } \mathrm{Pa}\end{array}$ \\
\hline $\bar{Y}$ & $\begin{array}{l}\text { measurement vector (random variable) of all means } \bar{Y}_{i} \quad(i= \\
1,2 . \mathcal{N} \text { ) with dimension } \mathcal{N}\end{array}$ \\
\hline$\mu$ & unknown true mean \\
\hline$\sigma$ & unknown true standard deviation \\
\hline$\sigma^{2}$ & unknown true variance \\
\hline$S$ & Standard deviation of a random variable \\
\hline$\tilde{S}_{i}$ & calc. standard deviation based on uncertainty, $\tilde{S}_{i}=u_{i} / 2$ \\
\hline $\mathbb{S}$ & standard deviation based on Monte Carlo Simulations \\
\hline$n$ & sample size for calculating a mean, e.g. $\bar{Y}_{i}$ \\
\hline$u$ & uncertainty, defined in NIST report 1297 [10] \\
\hline $\mathcal{N}$ & $\begin{array}{l}\text { number, e.g. general number, number of measurement points, } \\
\text { dimension of a vector }\end{array}$ \\
\hline $\mathcal{N}$ & Normal distribution \\
\hline$\chi^{2}$ & Chi-square distribution \\
\hline$p d f$ & probability density function \\
\hline dof & degree of freedom \\
\hline$J$ & Jacobian matrix in general \\
\hline$J$ & Jacobian matrix for the measurements \\
\hline$R D \%$ & $\begin{array}{l}\text { Relative Deviation in \%, vector containing all relative } \\
\text { deviations for all measuring points }\end{array}$ \\
\hline $\begin{array}{l}M R D \% \\
\max R D \%\end{array}$ & $\begin{array}{l}\text { Mean of Relative Deviation in \%, based on vector } R D \% \\
\text { maximum value of } M R D \%\end{array}$ \\
\hline Bias\% & Model Bias in \% \\
\hline$R k$ & Rank of the variance-covariance matrix of the parameters \\
\hline FitCap $\%$ & Fit Capability in \%, \\
\hline PreCap $\%$ & Predictive Capability in \% \\
\hline
\end{tabular}


Acknowledgment

The authors give their sincere thanks for valuable discussions to $\mathrm{Dr}$. Stefan Maixner (retired engineer from department of research, BASF SE, Germany).

Declaration of Competing Interest

The authors declare that they have no known competing financial interests or personal relationships that could have appeared to influence the work reported in this paper.

Supplementary materials

Supplementary material associated with this article can be found, in the online version, at doi:10.1016/j.fluid.2021.113374

\section{References}

[1] C. Muzny, Fitted Equations for Specific Properties, National Institute of Standards and Technology (NIST), 100 Bureau Drive, Gaithersburg, MD 20899, United States. 2022.

https://trc.nist.gov/TDE/Equations/FEquations.html

[2] A. Grybat, S. Horstmann, C. Ihmels, B. Schmid, Pure Component Equations -Fitting of Pure Component Equations Parameters- DDBSP-Dortmund Data Bank Software Package, DDBST - Dortmund Data Bank Software \& Separation Technology GmbH, Marie-Curie-Straße 10, 26129 Oldenburg, Germany, (2020) 49 .

http://www.ddbst.com/files/files/ddbsp/2020/Documentation/PCPEquationFit.p $\underline{\mathrm{df}}$

[3] A. Kud, C. Coquelet, S. Maixner, A new semi-empirical model for saturated vapor density of pure compounds, J. Chem. Eng. Data 65 (2020) 577-590. DOI: https://pubs.acs.org/doi/10.1021/acs.jced.9b00787

[4] I. Nezbeda, Simulations of vapor-liquid equilibria: routine versus thoroughness, J. Chem. Eng. Data 61 (2016) 3964-3969. D0I: https://doi.org/10.1021/acs.jced.6b00539

[5] B.L. Eggimann, Y. Sun, R.F. DeJaco, R. Singh, M. Ahsan, T.R. Josephson and J.I. Siepmann, Assessing the quality of molecular simulations for yapor-liquid equilibria: An analysis of the TraPPE Database, J. Chem. Eng. Data 65 (2020) 1330-1344. DOI: https://doi.org/10.1021/acs.jced.6b00539

[6] L. Riedel, Eine neue universelle Dampfdruckformel Untersuchungen über eine Erweiterung des Theorems der übereinstimmenden Zustände. Teil I. Chemie Ingenieur Technik, 26 (1954) 83-89. https://doi.org/10.1002/cite.330260206 
[7] J.W. Hogge, N.F. Giles, T.A. Knotts, R.L. Rowley, W.V. Wilding, The Riedel vapor pressure correlation and multi-property optimization, Fluid Phase Equilibria 429 (2016) 149-165.

http://dx.doi.org/10.1016/j.fluid.2016.08.032

[8] R.J. Larsen, M.L. Marx, An Introduction to Mathematical Statistics and Its Applications. 2nd ed. Prentice-Hall, New Jersey, 1986.

[9] G.A.F. Seber, C.J. Wild, Nonlinear Regression, Wiley Series in Probability and Mathematical Statistics, John Wiley \& Sons, New York, Chichester, Brisbane, Toronto, Singapore, 1988.

[10] B.N. Taylor, C.E. Kuyatt, Guidelines for Evaluating an Expressing the Uncertainty of NIST Measurement Results, National Institute of Standards and Technology, NIST Technical Note 1297, 1994 Edition. https://nvlpubs.nist.gov/nistpubs/Legacy/TN/nbstechnicalnote1297.pdf

[11] R.A. Fisher, On the mathematical foundations of theoretical statistics, Philosophical Transaction of the Royal Society A (1922) 309-368. https://doi.org/10.1098/rsta.1922.0009

[12] Mathcad 15, Software for mathematical modeling. PTC Inc., 121 Seaport Blvd, Boston, Massachusetts 02210 , USA.

[13] J.G. Kushmerick, Fundamental Physical Constants - NIST Standard Reference Database 121 - Physical Measurement Laboratory at National Institute of Standards and Technology (NIST), 100 Bureau Drive, Gaithersburg, MD 20899, United States. 2022.

https://physics.nist.gov/cuu/Constants/Table/allascii.txt

[14] E.W. Lemmon, R. Span, Short Fundamental Equations of State for 20 Industrial Fluids, Journal of Chemical \& Engineering Data 51 (2006) 785850. https://doi.org/10.1021/je050186n

[15] P.M. Mathias and T.W. Copeman, Extension of the Peng-Robinson Equation-of-state To Complex Mixtures: Evaluation of the Various Forms of the Local Composition Concept, Fluid Phase Equilibria 13 (1983) 91-108. https://doi.org/10.1016/0378-3812(83)80084-3

Data References

Database Fluoromethane Difluoromethane.xlsx 
Supplementary Material: Improvements for a Fully Consistent Description of the New Semi-Empirical Vapor Density Model for Pure Compounds

\author{
Alexander $\mathrm{Kud}^{\mathrm{a},{ }^{*}}$, Christophe Coquelet ${ }^{\mathrm{b}, \mathrm{c}}$ \\ * Corresponding author, Germany, E-mail: alexander.e.kud@yandex.com \\ ${ }^{a}$ retired Physical Chemist from Department of Research, BASF SE, Germany \\ bMines ParisTech, PSL University, CTP - Centre of Thermodynamics of \\ Processes, 35 rue Saint Honoré, Fontainebleau Cedex 77305, France \\ 'Université de Toulouse, IMT Mines Albi, UMR CNRS 5302, Centre Rapsodee, \\ Campus Jarlard, 81013 Albi CT Cedex 9, France \\ E-mail: christophe.coquelet@mines-albi.fr
}

\title{
Supplementary Material (SupMat)
}

In this work and for the simulations physical properties (constants), parameters and other quantities are considered. It is well known that in nonlinear modeling smallest deviations of these quantities can cause large deviations in the results. In order to have identical results between the different working groups and the same exactness, it is important to consider numerical values with many digits. 
SupMat A. Properties of fluoromethane and difluoromethan

Table A

Physical properties of fluoromethane and difluoromethane used for the calculations in this work

\begin{tabular}{|c|c|c|c|c|}
\hline Property & $\begin{array}{c}\text { Fluoromethane } \\
\text { (R41) }\end{array}$ & References & $\begin{array}{c}\text { Difluoromethane } \\
\text { (R32) }\end{array}$ & References \\
\hline Molar mass $\mathrm{kg} / \mathrm{mol}$ & 0.034033217 & [A.1] & 0.052023694 & [A.1] \\
\hline Critical temperature / K & 317.454 & [A.2] & 351.2812 & [A.2] \\
\hline Critical pressure / $\mathrm{Pa}$ & 5881059.5 & [A.2] & 5784146.5 & [A.2] \\
\hline Critical density / $\mathrm{kg} / \mathrm{m}^{3}$ & 307.965042 & [A.3] & 425.1621758 & [A.3] \\
\hline Acentric factor & 0.2004 & [A.5] & 0.2769 & [A.6] \\
\hline Triple point temperature / K & 129.82 & {$[A .5,7]$} & 136.34 & [A.6] \\
\hline Critical compressibility factor ${ }^{1)}$ & 0.246231 & & 0.242324 & \\
\hline Compressibility factor for vapor ${ }^{2)}$ at $T_{\text {tp }}$ & 0.999544 & {$[A .5,7]$} & 0.999913 & [A.6] \\
\hline
\end{tabular}

1) calculated from critical properties. 2) calculated from FEoS in REFPROP 10.0

Physical constants, such as universal gas constant, can be found in the special table from NIST [A.8].

\section{SupMat B Data treatment}

An EXCEL file is provided for all experimental data that have been considered in this work, checked and cleared of outliers. The first column represents the independent variable (e.g., mean temperature), the second column indicates the mean of the measured value of the dependent variable, the third column indicates the standard deviation of the mean of the dependent variable, the fourth indicates the covariance between the measured mean of the dependent and independent variables, and the fifth column indicates the sample size for the measurement of the dependent variable. The databases for the evaluation are provided and are listed in "Data References" in the article.

SupMat B.1 Fluoromethane (R41)

\section{B.1.1 Vapor pressure data}

The raw experimental data were obtained from the ref [A.3]. The data were treated and analyzed as follows:

-All data points with the remark "not accepted" were removed.

-The calculated standard deviations $\tilde{S}_{i}$ can be figured out from the uncertainty data according to $\tilde{S}_{i}=u_{i} / 2$. Then the Coefficient of Variation (CoV) 
is calculated from the mean of experimental data according to $\operatorname{CoV}_{i}=\tilde{S}_{i} / \bar{Y}_{i}$. A variance and CoV analysis show the following results:

- There are homogeneous and heterogeneous variances.

- There is a homogeneous and heterogeneous structure in CoV.

- There are unrealistically small CoV values $\left(3.3 \cdot 10^{-5}\right)$ that most likely relate to apparatus uncertainties and not to uncertainties of a whole experiment replicate.

- There are most likely also assumed and no calculated uncertainties.

- There is most likely also calculated vapor pressure data.

- There are measurement data whose uncertainties or standard deviations are much larger than the measured vapor pressure.

Experimental data with unrealistically small CoV values were replaced by a realistic CoV value of about 0.008 . This value is based on a CoV analysis of experimental vapor pressure data and experimental pressure data at the critical point and agrees very well with experience values for pressure data over a wide temperature range. A mean sample size $\tilde{n}$ of about 17 can be estimated from the student $t$ distribution (see eq (2D-8) in supMat 2D in ref [3]). In this way, standard deviation and sample size can be estimated. We are aware that, strictly speaking, this is not possible because of an underdetermined equation system. But with plausible, practical assumptions, a good estimate of an unknown mean standard deviation and mean sample size can be made. In this way, parameter estimation with standardized residuals becomes possible.

The next step is to detect the outliers. For this purpose, it is assumed that the Riedel vapor pressure equation [7], also known as the DIPPR 101 equation, is the "true" function describing the vapor pressure data, since it describes the vapor pressure data very well compared to other known equations (see list of equations in ref [2]).

Since the raw data set has vapor pressure data near the triple point temperature, it will be checked especially for outliers. For this purpose, the vapor pressure calculated with FEoS [A.5] is assumed to be very trustworthy with REFPROP 10.0 at the triple point. Therefore, the first parameter estimation is calculated according to formulation problem (PE 1) with constraint on the triple point pressure. All vapor pressure data whose relative deviations are greater than $0.5 \%$ in absolute value or whose weighted or standardized residuals are greater than two standard deviations are eliminated. For $\sim 75 \%$ of the 33 eliminated outliers, both exclusion criteria apply together. The remaining 25\% are excluded only by the relative error of $0.5 \%$. Now, the database for the parameterization consists of 293 experimental data points.

If a residuals analysis of the database shows stochastic deviations, then random variables are present in the experimental data and the database can be used directly for parameterization. If, however, systematic deviations 
are recognizable in the residuals, as e.g. Fig. E.5 shows, then correlated data are present which have been calculated with equations. The sum of the weighted correlated residual squares $(S W S)$ calculated in this way is not $\chi^{2}$ distributed. However, the test quantity SWS should have the character of a $\chi^{2}$ distributed quantity as much as possible. This can be achieved with a pocketknife trick by calculating a mean CoV $(\widetilde{\mathrm{CoV}})$ for all experimental data points such that the test quantity $S W S$ is $\chi^{2}$ distributed, knowing full well that this is not the case. This correction, described below, can only partially improve so that the parameterization algorithm computes the most likely solution of a $\chi^{2}$ distributed quantity. A clever transformation of the general definition equation for computing the SWS, together with the assumptions $S_{i} \cong \tilde{S}_{i}$ and $\tilde{n}:=n_{i}$, yields the equation (B.1)

$\operatorname{SWS}\left(\bar{Y}_{p}, \tau, \widetilde{C O V}, \tilde{n}, \Theta_{p}\right)=\frac{\tilde{n}}{\overline{\mathrm{COV}}^{2}} \sum_{i=1}^{\mathcal{N}_{p}}\left(\frac{\bar{Y}_{p i}-F_{p}\left(\tau_{i}, \Theta_{p}\right)}{\bar{Y}_{p i}}\right)^{2}=\frac{\tilde{n}}{\overline{\operatorname{CV}}^{2}} \operatorname{SRD}\left(\bar{Y}_{p}, \tau, \Theta_{p}\right)$.

The sum $S R D$ in (B.1) is the well-known objective function of the relative residuals and is easy to calculate. The most likely value of the probability density function $p d f_{\chi^{2}}$ of the $\chi^{2}$ distribution is the maximum $p d f_{\chi^{2}, \max }$. The quantile $\chi_{\max }^{2}$ for this maximum can be calculated according to the simple equation $\chi_{\text {max }}^{2}=d o f-2=\mathcal{N}_{p}-\mathcal{N}_{\Theta_{p}}-2$ and is identical to $\operatorname{s} W S\left(\bar{Y}_{p}, \tau, \widetilde{C o V}, \tilde{n}, \Theta_{p}\right)$ (see chapter 7.5 in ref [8] or other textbooks of statistics). The mean ( $\widetilde{C O V}$ ) is thus given by the equation (B.2)

$\widetilde{C O V}=\sqrt{\frac{\tilde{n} \operatorname{SRD}\left(\bar{Y}_{p}, \tau, \Theta_{p}\right)}{\mathcal{N}_{p}-\mathcal{N}_{\Theta_{p}}-2}}$.

Equation (B.2) can be used to calculate a mean standard deviation $\tilde{S}_{i}=\widetilde{C o V}$. $\bar{Y}_{p i}$ for each experimental data point. The new objective function is given by $\mathrm{Eq} .(\mathrm{B} \cdot 3)$.

$S W S_{1}\left(\bar{Y}_{p}, \tilde{S}, \tau, \Theta, \tilde{n}\right)=\tilde{n} \sum_{i=1}^{N}\left(\frac{\bar{Y}_{p i}-F_{p}\left(\tau_{i}, \Theta_{p}\right)}{\tilde{S}_{i}}\right)^{2}$.

In the next iteration, the sample size $\tilde{n}$ is checked with the quantile of the maximum of the $p d f_{\chi^{2}, \max }$. For this purpose, the quotient $\lambda$ is calculated according to the equation (B.4)

$\lambda=\frac{s W s_{1}}{\chi_{\max }^{2}}$.

If $\lambda$ differs significantly from 1 (about $\pm \odot .05$ ), then a corrected sample size $n^{*}$ is calculated according to the equation (B.5)

$n^{*}=\frac{\tilde{n}}{\lambda}$.

The new objective function is given by Eq. (B.6)

$S W S_{2}\left(\bar{Y}_{p}, \tilde{S}, \tau, \Theta, n^{*}\right)=n^{*} \sum_{i=1}^{N}\left(\frac{\bar{Y}_{p i}-F_{p}\left(\tau_{i}, \Theta_{p}\right)}{\tilde{S}_{i}}\right)^{2}$ 
and will be used for MCS. For fluoromethane, this approach calculates a mean relative error $\widetilde{C o V} \%$ for all experimental data of about $1.45 \%$ from the triple point temperature to the critical temperature, and the mean sample size $n^{*}$ is about 23 .

of course, $S W S_{2}$ can no longer be used as an evaluation variable, since the statistics of the $\chi^{2}$ distribution have already been plugged in as information to use an optimal $\chi^{2}$ estimator as an objective function. Thus, the parameterization is based on equation (B.6) and the evaluation is based on relative deviations $\mathrm{RD} \%$ using the $S R D$ objectiv function.

\section{B.1.2 Vapor density data}

The raw data [B.2], [B.3] consist of a total of 18 measured data. From the experimental data of Cawood and Patterson [B.3] a mean CoV of 0.02534 can be calculated from experiment repetitions at four temperatures (303.163, $308.162,315.139,316.139) \mathrm{K}$. The experimental data point at $314.425 \mathrm{~K}$ is an outlier and is removed. From the experimental vapor density data, the compressibility factors were calculated using the vapor pressure equation and compared with the compressibility factors of the FEoS in REFPROP 10.0 as a function of absolute temperature. It is noticeable that the experimental data point at $269.1 \mathrm{~K}$ from ref [B.2] is significantly too small, which means that the vapor density is significantly too large. This experimental data point was also removed. The experimental data data base consists of 16 experimental data points from the temperature range $T \in[291.172,317.119] \mathrm{K}$ or $\tau \in$ $[0.917,0.9989]$. These data are not sufficient for parameterization of the vapor density model (8) over the entire temperature range. Therefore, vapor density data are simulated with trustworthy EoS in the temperature range $T \in\left[\mathrm{T}_{t p}, 290\right] \mathrm{K}$. The following EoS were selected for this purpose:

1. FEoS implemented in Refprop10 [A.5]

2. Peng Robinson EoS [B.4] with Mathias Copeman (MC) a function [B.5]

3. Peng Robinson with a function based on Acentric Factor $\dot{\omega}$ [B.4]

4. modified PT EoS with MC a function [B.6]

With each EoS, 16 data points were simulated. The relative error is assumed to be twice as large as for the experiments. This corresponds to a factor of 4 in the variance. The four EoS should be weighted together with the same variance as the experiments, hence the doubling in standard deviation.

To get an idea of how well the vapor densities at the triple point are described by known EoS, Table B.1 lists the calculated vapor densities that agree very well. 
Table B.1

Calculated fluoromethane (R41) vapor density e from different EoS at triple point temperature

\begin{tabular}{ll}
\hline & $\mathrm{e} / \mathrm{kg} / \mathrm{m}^{3}$ \\
\hline FEoS in REFPROP 10.0 & 0.010888 \\
PR EoS (MC) & 0.010882 \\
PR EoS $(\dot{\omega})$ & 0.010882 \\
PT EoS $(\omega)$ & 0.010882 \\
Modified PT EoS (MC) & 0.010887 \\
\hline Mean & 0.010884 \\
Ideal Gas for comparison ${ }^{1)}$ & 0.010879 \\
\hline
\end{tabular}

1) at saturated pressure

SupMat B.2 Difluoromethane (R32)

\section{B.2.1 Vapor pressure}

To the raw database [A.3] of 766 data points, the triple point pressure calculated with FEoS in REFPROP 10.0 is also added, since there is no measured data near the triple point temperature. After removing the outliers and the data treatment (described in Section B.1.1). The database consists of 716 data points obtained after data treatment and removing of the outliers. A mean relative experimental error of $\widetilde{\mathrm{COV}}$ is estimated to be 0.0161 and a mean sample size of 30 from the triple point temperature to the critical temperature are estimated.

\section{B.2.2 Vapor density}

The raw data base was collected by four working groups [B.8 to B.11] in the temperature range $T \in[253.07,351.263] K$ and reduced temperature $\tau \in[0.72,0.9999]$, respectively. A literature search yielded additional measured data from Defibaugh [B.12] in the temperature range of $T \in[220,351] K$, which were used to parameterize an MBWR EoS [B.14]. This EoS was used to calculate vapor densities, which can be found in Table 8 in ref [B.12]. An analysis shows that the MBWR EoS parametrized with Defibaugh data predicted density in a very good agreement with experimental data from [B.8-11]. Of these data, only those in the temperature range of $T \in[220,253] K$ or $\tau \in[0.63,0.72]$ were selected to complete the above temperature range $\tau \in[0.72,0.9999]$.

Since the available experimental vapor density data do not cover the necessary temperature range from the triple point to the critical temperatures, three equations of state with different alpha functions are used for testing in the temperature range $T \in\left[T_{t p}, 253\right] K$ or $\tau \in\left[\tau_{t p}, 0.72\right]$. The tests consist of the selection of the more suitable EoS for this purpose. Selection criterion for extrapolation of EoS outside the temperature measurement range shall be 
the smallest vapor density deviation in the temperature range of the measurements.

The following EoS are used for the test.

a) FEOS in REFPROP $10.0[$ [A.6].

b) PR EoS with acentric factor [B.4].

c) PR EoS with MC a function [B.5].

d) Patel Teja Eos with acentric factor [B.13].

e) Modified PT with MC a function [B.5].

The FEoS shows the lowest vapor density deviations in the measurement range and is therefore selected for the extrapolation of simulated experimental data. Table B.2 lists the calculated vapor densities at the triple point temperature.

Table B.2

Calculated difluoromethane (R32) vapor density $\varrho$ from different EoS at triple point temperature.

\begin{tabular}{lc}
\hline & $\mathrm{e} / \mathrm{kg} / \mathrm{m}^{3}$ \\
\hline FEoS in REFPROP 10.0 & 0.0022033 \\
PR EoS (MC) & 0.0022030 \\
PR EoS $(\omega)$ & 0.0022030 \\
PT EoS $(\dot{\omega})$ & 0.0022030 \\
Modified PT EoS (MC) & 0.0022030 \\
\hline Mean & 0.0022030 \\
Ideal Gas for comparison $^{1)}$ & 0.0022029 \\
\hline
\end{tabular}

1) at saturated pressure

SupMat C Definition of the assessment quantities and Monte Carlo Simulations

The NIST database has an experimental mean value and an associated uncertainty for each measured temperature [C.1]. The specification of the uncertainty is based on an infinitely large sample size [C.1]. A standard deviation can be calculated from the uncertainty. However, this standard deviation no longer has a stochastic character, since this has been lost by error propagation calculations, e.g. with equations related to the experimental device. However, stochastic standard deviations are needed for the weighted parameterization. For this reason, random standard deviations are generated using Monte Carlo simulations (see Appendix 2D of the Supporting Information in reference [3]). It should be mentioned that the experimental average 
values of the database don't absolutely change. These values are of course directly used for parameterization.

Based on the MCS, not only the mean values of the estimated parameters and the assessment quantities $M R D \%, \max R D \%$ and $B i a s \%$ are obtained, but also their standard deviations. Thus, statements about their certainty or uncertainty can also be made.

For simplification, the abbreviation $U=p, \varrho$ will be used for the state variables vapor pressure and vapor density.

The coefficient of variation $\mathrm{CoV}$ and the standard deviation $S$ belong to the evaluation of the results, are detailed forthe parameters and the evaluation variables in the context of the MCS.

\section{The Parameters}

$\widehat{\Theta}_{U, j, \kappa}$ denotes the estimated parameter $j$ for the state variable $U$ at MCS loop number $\kappa$. The average parameter vector $\bar{\Theta}_{U}$ is calculated according to Eq. (C.1)

$\bar{\Theta}_{U, j}=\frac{1}{\mathcal{N}_{M C S}} \sum_{\kappa=1}^{\mathcal{N}_{M C S}} \widehat{\Theta}_{U, j, K}$.

The Coefficient of Variation in per cent (CoV\%) is calculated as follows (Eq. (C.2))

$\operatorname{CoV} \%\left(\bar{\Theta}_{U, j}\right)=\frac{\operatorname{stdev}\left(\bar{\Theta}_{U, j}, \widehat{\Theta}_{U, j, \kappa} \quad \kappa=1,2 \ldots \mathcal{N}_{M C S}\right)}{\left|\bar{\Theta}_{U, j}\right|} \cdot 100 \%$,

where stdev denotes the known equation for calculation of the standard deviation.

The Fit Assessment Quantities

The Relative Deviation\% $\left(R D \%(U)_{i, \kappa}\right)$ for measurement No. $i$ at $T_{i}$ in the Monte Carlo iteration number $\kappa$ is calculated according to Eq. (C.3)

$R D \%(U)_{i, \kappa}=\frac{\bar{Y}_{U, i}-\widehat{U}_{i \kappa}}{\bar{Y}_{U, i}} \cdot 100 \%$,

where $\widehat{U}_{i \kappa}$ is the estimated vapor state variable which is identical to Eq. (C.4)

$\widehat{U}_{i \kappa} \equiv F_{U}\left(T_{i}, \widehat{\Theta}_{U, \kappa}\right)$

and $\bar{Y}_{U, i}$ denotes the experimental mean at $T_{i}$. The average Relative Deviation\% at $T_{i}$ for all MCS is defined by Eq. (C.5)

$\overline{R D \%}(U)_{i}=\frac{1}{\mathcal{N}_{M C S}} \sum_{\kappa=1}^{\mathcal{N}_{M C S}} R D \%(U)_{i, \kappa}$.

This quantity is used to calculate the graphs for the diagrams. The Mean Relative Deviation\% $M R D \%(U)_{\kappa}$ for the $\kappa^{\text {th }}$ iteration is defined by Eq. (C.6) 
$M R D \%(U)_{\kappa}=\frac{100 \%}{\mathcal{N}_{U}} \sum_{i=1}^{\mathcal{N}_{U}}\left|\frac{\bar{Y}_{U, i}-\widehat{U}_{i \kappa}}{\bar{Y}_{U, i}}\right|=\frac{100 \%}{\mathcal{N}_{U}} \sum_{i=1}^{\mathcal{N}_{U}}\left|R D \%(U)_{i, \kappa}\right|$

where $\mathcal{N}_{U}$ is the number of all experimental data for the state variable $U$. The average $\overline{M R D \%}$ over all numbers $\mathcal{N}_{M C S}$ is defined by Eq. (C.7)

$\overline{M R D \%}(U)=\frac{1}{\mathcal{N}_{M C S}} \sum_{\kappa=1}^{\mathcal{N}_{M C S}} \operatorname{MRD} \%(U)_{\kappa}$.

The standard deviation $\mathbb{S}$ of $M R D \%(U)$ gives information about the mean deviation of $M R D \%(U)_{\kappa}$ and is calculated according to the known equation for standard deviation which is written here in abbreviated form (Eq. (C.8))

$\mathbb{S}_{M R D \%(U)}=\operatorname{stdev}\left(\overline{M R D \%}(U), M R D \%(U)_{\kappa} \quad \kappa=1,2 \ldots \mathcal{N}_{M C S}\right)$.

In context with the average relative deviation, the maximum of the absolute relativeof the vector element in the vector $\overline{R D \%}(U)$ should be included as an additional assessment variable. This quantity is more sensitive to outliers in the data base or to an extreme "model bias" than the average $\overline{M R D \%}(U)$. It is defined for the iteration $\kappa$ as follows (Eq. (C.9))

$\max R D \%(U)_{\kappa}=\max \left[\left|R D \%(U)_{i, \kappa}\right| \quad i=1,2 \ldots \mathcal{N}_{U}\right]$.

The average of all MCS can be calculated according to the Eq. (C.10)

$\overline{\max R D \%}(U)=\frac{1}{\mathcal{N}_{M C S}} \sum_{\kappa=1}^{\mathcal{N}_{M C S}} \max R D \%(U)_{\kappa}$.

The calculation of a standard deviation for an extreme value $\overline{M A X \%}(U)$, which is always at the same experimental temperature in case of a systematic deviation for each MCS, is no longer random. Since there are unfortunately not only random variables in a experimental data set, but also mean values simulated with models, such a value can lead to a systematic deviation at always the same measurement temperature. Therefore, the specification of a standard deviation is not recommended.

The model bias for iteration $\kappa$ is defined as follows (Eq. (C.11))

$\operatorname{Bias} \%(U)_{\kappa}=\frac{100 \%}{\mathcal{N}_{U}} \sum_{i=1}^{\mathcal{N}_{U}} \frac{\bar{Y}_{U, i}-\widehat{U}_{i \kappa}}{\bar{Y}_{U, i}}$

and the corresponding average quantity and standard deviation are as expected ((Eqs. (C.12 and C.13))

$\overline{\operatorname{Blas} \%}(U)=\frac{1}{\mathcal{N}_{M C S}} \sum_{\kappa=1}^{\mathcal{N}_{M C S}} \operatorname{Bias} \%(U)_{\kappa}$,

$\mathbb{S}_{\text {Bias } \%(U)}=\operatorname{stdev}\left(\overline{\operatorname{Blas} \%}(U), \operatorname{Bias} \%(U)_{\kappa} \quad \kappa=1,2 \ldots \mathcal{N}_{M C S}\right)$.

In this context, characteristic quantities such as boiling temperature, triple point pressure or vapor density at the triple point can also be determined using MCS. 


\section{The Physical Quantities}

The boiling point temperature $\widehat{T}_{b}$ is calculated from average estimated parameter vector $\bar{\Theta}_{p}$ according to the implicit vapor function $F_{p}$ (Eq. (C.14))

$F_{p}\left(\widehat{T}_{b}, \bar{\Theta}_{p}\right)-p_{0}=0$

where $p_{0}$ is the standard pressure at 1 atm $\left(p_{0}=1.01325 \cdot 10^{5} \mathrm{~Pa}\right)$. The corresponding standard deviation is calculated by solving the implicit Eq. (C.15)

$F_{p}\left(T_{b, \kappa}, \widehat{\Theta}_{p, \kappa}\right)-p_{0}=0$

for the boiling point at each temperature $T_{b, \kappa}$ where the average boiling temperature from all MCS is as follows (Eq. (C.16))

$\bar{T}_{b}=\frac{1}{\mathcal{N}_{M C S}} \sum_{\kappa=1}^{\mathcal{N}_{M C S}} T_{b, \kappa}$.

From this, the mean deviation (standard deviation) $\mathbb{S}_{T_{b}}$ around the estimated mean $\bar{T}_{b}$ can be calculated by Eq. (C.17)

$\mathbb{S}_{T_{b}}=\operatorname{stdev}\left(\bar{T}_{b}, T_{b, \kappa} \quad \kappa=1,2 \ldots \mathcal{N}_{M C S}\right)$.

Since the average $\bar{T}_{b}$ is almost identical to the estimated $\widehat{T}_{b}$, this is also the standard deviation for the estimated $\widehat{T}_{b}$ ((Eq. (C.18))

$\mathbb{S}_{\widehat{T}_{b}} \approx \mathbb{S}_{T_{b}}$

The boiling point density for vapor is calculated using the average of estimated parameter according to the Eq. (C.19)

$\hat{\varrho}_{b}=F_{\varrho}\left(\widehat{T}_{b}, \bar{\Theta}_{p}, \bar{\Theta}_{Z}\right)$.

The standard deviation $\mathbb{S}_{\widehat{Q}_{b}}$ is calculated similar to $\mathbb{S}_{T_{b}}$ and the equations are as follows (Eqs. (C.20 to C.22))

$\varrho_{b, \kappa}=F_{Q}\left(\tau, \widehat{\Theta}_{p, \kappa}, \widehat{\Theta}_{z, \kappa}\right)$

$\bar{\varrho}_{b}=\frac{1}{\mathcal{N}_{M C S}} \sum_{\kappa=1}^{\mathcal{N}_{M C S}} \varrho_{b, \kappa}$

$\mathbb{S}_{\widehat{\varrho}_{b}} \approx \operatorname{stdev}\left(\bar{\varrho}_{b}, \varrho_{b, \kappa} \quad \kappa=1,2 \ldots \mathcal{N}_{M C S}\right)$.

The triple point pressure and its corresponding density value are calculated directly from the model equations according to Eqs. (C.23 and C.24)

$\hat{p}_{t p}=F_{p}\left(\bar{T}_{t p}, \bar{\Theta}_{p}\right)$

$\hat{\varrho}_{t p}=F_{\varrho}\left(\bar{T}_{t p}, \bar{\Theta}_{p}, \bar{\Theta}_{Z}\right)$.

It is not recommended to calculate the stdev of $\hat{p}_{t p}$ and $\hat{\varrho}_{t p}$ considering MCS because the optimization algorithm reaches the limit of the constraint ( $\bar{p}_{t p}$ or $\bar{\varrho}_{t p}$ ) or is in a range very close to this limit. In effect, the standard deviation is a random variable and close to a constraint value the optimiza- 
tion algorithm uses penalty terms that destroy the random character of a variance.

The determination of the minimum number of MCS is based on the statistics for control charts for the estimated parameters. For each parameter, averages of 1000 MCS are calculated for such a control chart, i.e. a block consists of 1000 MCS. A confidence range is calculated based on the student $t$ probability density function. Experiences show that a confidence interval of 0.809 is strict to detect and abort convergence. At least 15 blocks must be calculated at the beginning of the MCS. The observation space for the current mean calculation is 10 blocks retrospectively. Within 10 blocks the parameter value must not exceed or fall below the confidence limits. If this condition is met, the MCS is aborted.

SupMat D Estimated Parameter Values for Simulations

Table D provides the average values of estimated parameter with more digits for simulations. Results are based on MCS.

Table D

Average values of estimated parameter with more digits for simulations.

\begin{tabular}{crr}
\hline & \multicolumn{2}{c}{ Parameter values for } \\
Parameter & Fluoromethane (R41) & Difluoromethane (R32) \\
\hline $\bar{\Theta}_{z 1}$ & 1.626221818488 & 3.938050095271 \\
$\bar{\Theta}_{z 2}$ & 0.234926702594 & 0.439081284486 \\
$\bar{\Theta}_{z 3}$ & 0.944612459040 & 0.895572259879 \\
$\bar{\Theta}_{z 4}$ & 4.249065063034 & 3.136381522713 \\
$\bar{\Theta}_{z 5}$ & 1.824148272542 & 1.796494026212 \\
$\bar{\Theta}_{z 6}$ & 0.555004927438 & 0.660228583214 \\
$\bar{\Theta}_{p 1}$ & 3023.800649449500 & 3933.332225338400 \\
$\bar{\Theta}_{p 2}$ & -5.976977380797 & -8.105025423986 \\
$\bar{\Theta}_{p 3}$ & 1.567545795817 & 1.638388300915 \\
$\Theta_{p 4}$ & 2 & 2 \\
$\mathcal{N}_{Z}$ & 2 & 2 \\
\hline
\end{tabular}

We use the following parameter vectors as guess values for a successful parameterization of the $\mathrm{Z}$ model (Eq. (D.1))

$\Theta_{Z, \text { ini }}:=\left\{\begin{array}{lllll}\left(\begin{array}{lllll}2.6 & 0.5 & 0.95\end{array}\right)^{\mathcal{T}} & \text { if } \mathcal{N}_{z}=1 \\ \left(\begin{array}{lllllll}1.5 & 0.2 & 0.95 & 2.5 & 0.53 & 0.5\end{array}\right)^{\mathcal{T}} & \text { if } \mathcal{N}_{z}=2\end{array}\right.$.

Parameter estimation with one term converges very quickly. The recommended parameter vectors for two terms $\left(\mathcal{N}_{z}=2\right)$ are very robust. If problems still 
occur, another proposal can be tested for success. An equation for testing the necessary precondition of convergence capability in the case of nonlinear parameter estimation problems is given from Marquardt (see page 439 in [D.1]) as an example. More recent findings in this area are described for example in the work of Clesse et al. [D.2].

For parameter estimation and calculation of the cov matrix of parameters, a specially scaled form $f_{p}^{*}$ (Eq. (D.2)) is used instead of the vapor pressure function given by eq (6). This ensures that the values of the parameters are in the numerical range of $[0.1,10]$. The calculation does not fail. The scaled form is given by Eq. (D.2).

$f_{p}^{*}\left(\tau, T_{c}, \Theta_{p}\right)=\exp \left[\frac{\Theta_{p 1} \cdot 1000}{T_{c}}\left(1-\frac{1}{\tau}\right)+\Theta_{p 2} \cdot 10 \cdot \ln (\tau)+\Theta_{p 3} \cdot\left(\tau^{\Theta_{p 4}}-1\right)\right]$.

With the initial parameter $\Theta_{p, i n i}:=\left(\begin{array}{lll}3.5 & -0.781 .83\end{array}\right)^{\mathcal{T}}$, every compound converged so far. The results of the parameter estimation are converted and given for Eq. (6).

SupMat E Supplementary results

Table E.1

Estimated parameter for difluoromethane (R32). Results are based on MCS.

\begin{tabular}{|c|c|c|c|c|c|c|c|}
\hline \multirow{2}{*}{$\begin{array}{l}\text { Estimated } \\
\text { Parameter }\end{array}$} & \multicolumn{2}{|c|}{$\mathrm{PE} 1^{1)}$} & \multicolumn{2}{|c|}{$P E 2^{2)}$} & \multicolumn{2}{|c|}{$\mathrm{PE} 3^{3)}$} & \multirow{2}{*}{$\begin{array}{l}\text { Remarks on the Resposibility } \\
\text { and Meaning of Parameters }\end{array}$} \\
\hline & Average & CoV\% & Average & Cov\% & Average & CoV\% & \\
\hline $\bar{\theta}_{p 1}$ & 3933.2 & 0.022 & & & 3933.3 & 0.022 & \\
\hline $\bar{\Theta}_{p 2}$ & -8.105 & 0.065 & & & -8.105 & 0.065 & $\Theta_{p 4, o p t}=2$ \\
\hline $\bar{\theta}_{p 3} \times 10^{5}$ & 1.639 & 0.079 & & & 1.638 & 0.079 & \\
\hline $\bar{\theta}_{z 1}$ & & & 3.67 & 5.2 & 3.938 & 18.3 & for $T_{c}$ region \\
\hline $\bar{\theta}_{z 2}$ & & & 0.438 & 1.5 & 0.439 & 1.4 & for $T_{c}$ region \\
\hline $\bar{\theta}_{z 3}$ & & & 0.895 & 0.4 & 0.896 & 0.5 & for ideal gas temperature ${ }^{4)}$ \\
\hline $\bar{\theta}_{z 4}$ & & & 3.134 & 1.6 & 3.136 & 1.7 & for $T_{\text {tp }}$ region \\
\hline $\bar{\Theta}_{z 5}$ & & & 1.794 & 6.2 & 1.796 & 5.8 & for $\mathrm{T}_{\mathrm{tp}}$ region \\
\hline $\bar{\theta}_{z 6}$ & & & 0.674 & 1.5 & 0.66 & 5.9 & Fraction of $\mathrm{T}_{\mathrm{c}}$ Region description \\
\hline
\end{tabular}

1) vapor pressure data only. 2) vapor density data only. 3) vapor pressure and vapor density data simultaneously.

For simulations much more digits are necessary, see appendix 4. 4) model specific temperature

The vapor pressure parameters for both evaluations according to formulation problems (PE 1) and (PE 3) are equal in a statistical sense. This can also be assumed for the $Z$ parameters, since the parameter $\bar{\Theta}_{z 1}$ is estimated with a much larger CoV for the evaluation according to formulation problem (PE 3 ) than in the case of a separate evaluation formulation problem (PE 2). The reason for this is unknown. In the case of difluoromethane (R32), the parameters $\bar{\Theta}_{z 3}$ and $\bar{\Theta}_{z 4}$ are described more precisely (lower values of CoV\%) than for fluoromethane $(\mathrm{R} 41) . \bar{\Theta}_{z 3}$ and $\bar{\Theta}_{z 4}$ parameters are necessary to rep- 
resent compressibility factor $Z$ in the temperature range near the triple point temperature. With R32, the dispersion of the vapor density data is not so large in comparison to R41's values.

Table E.2

Regression assessment for the different kind of parameter estimation formulations (PE 1) to (PE 3) for difluoromethane (R32). Results are based on MCS.

\begin{tabular}{lcccccc}
\hline & \multicolumn{2}{c}{ PE 11) } & \multicolumn{2}{c}{ PE 2) } & \multicolumn{2}{c}{ PE 33) } \\
Assessment Quantity & Average & Stdev & Average & Stdev & Average & Stdev \\
\hline MRD\% for vapor pressure & 0.171 & 0.0001 & & & 0.171 & 0.0002 \\
maxRD\% for vapor pressure & 0.513 & n.r. & & & 0.512 & n.r. \\
Bias\% for vapor pressure & -0.019 & 0.0021 & & -0.017 & 0.002 \\
& & & & & & \\
MRD\% for vapor density & & & 0.864 & 0.014 & 0.862 & 0.015 \\
maxRD\% for vapor density & & & 6.410 & n.r. & 6.391 & n.r. \\
Bias\% for vapor density & & & 0.0136 & 0.040 & -0.017 & 0.002 \\
\hline
\end{tabular}

1) vapor pressure data only. 2) vapor density data only. 3) vapor pressure and vapor density data simultaneously. n.r. is not recommended

In this case, there are no or only very slight differences in the ratings for the separate and simultaneous evaluations. Both evaluations give good ratings.

Table E.3

Comparison of the results of the physical properties of difluoromethane (R32) for the different parameter estimation formulations (PE 1) to (PE 3). Results are based on MCS.

\begin{tabular}{|c|c|c|c|c|c|c|c|}
\hline \multirow[b]{2}{*}{ Quantity } & \multirow[b]{2}{*}{ Database $^{4)}$} & \multicolumn{2}{|c|}{$\mathrm{PE} 1^{1)}$} & \multicolumn{2}{|c|}{$P E 2^{2)}$} & \multicolumn{2}{|c|}{ PE $3^{3)}$} \\
\hline & & Average & Stdev & Average & Stdev & Average & Stdev \\
\hline Boiling point temperature / K & 221.38 & 221.42 & 0.001 & & & 221.43 & 0.001 \\
\hline Boiling point temperature $/{ }^{\circ} \mathrm{C}$ & -51.78 & -51.73 & 0.001 & & & -51.72 & 0.001 \\
\hline Boiling point vapor density $/ \mathrm{kg} / \mathrm{m}^{3}$ & 2.988 & & & 2.988 & 0.001 & 2.985 & 0.001 \\
\hline Triple point vapor pressure / $\mathrm{Pa}$ & 47.99983 & 48.05325 & n.r. & 48.05328 & 6) & 48.03778 & n.r. \\
\hline Triple point vapor density / kg/m³ & 0.002203 & & & 0.002205 & n.r. & 0.002205 & n.r. \\
\hline Compressibility factor at triple point & 0.999917 & 1.001026 & 5) & 0.999917 & n.r. & 0.999920 & n.r. \\
\hline "Ideal gas temperature" / K & & & & & 1.98 & 122.10 & 0.60 \\
\hline
\end{tabular}

1) vapor pressure only. 2) vapor density only. 3) vapor pressure and vapor density data simultaneously.

4) calc with the FEoS in REFPROP 10.0, except $T_{b}$ which is a mean from measurements. 5) calculated

$Z_{\mathrm{tp}}$ from $\mathrm{p}_{\mathrm{tp}}(\mathrm{PE} 1)$ and vapor density (database). 6) vapor pressure calculated from compressibility factor and vapor density both from (PE 2). n.r. not recommended. 
The conclusions obtained from the results in Tab. E.3 are basically the same for difluoromethane (R32) as for fluoromethane.

For the vapor density calculations in Fig. E.4, different EoS were parameterized using the MC alpha function. The following parameter vectors were used for this purpose:

$M C_{\text {mod.PT Eos }}=\left(\begin{array}{lll}0.28058954157867 & 0.52602551436449 & -0.476614689389163\end{array}\right)^{\mathcal{T}}$

$Z_{C}(\bmod . P T E o S)=0.257140715165181$.

$M C_{P R E O S}=\left(\begin{array}{lll}0.702756 & -0.269279 & 0.384198\end{array}\right)^{\mathcal{T}}$

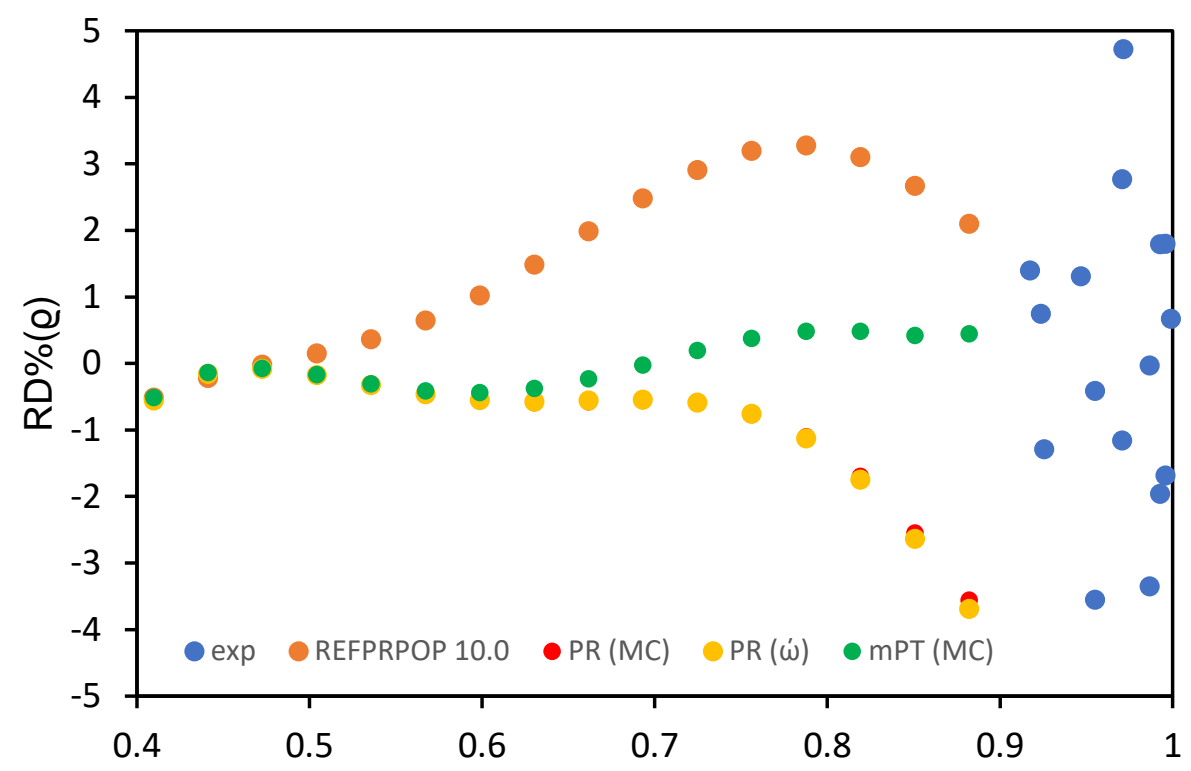

Reduced Temperature

Fig. E.4. Average Relative Deviation\% $(\overline{R D \%}(\varrho))$ for vapor density of fluoromethane (R41) vs. reduced temperature from the triple point temperature $\left(\tau_{t p}=0.409\right)$ to the critical temperature. The evaluation is based on formulation problem (PE 3$). \overline{R D \%}(\varrho)$ are calculated from the semi-empirical vapor density model and the following density data used for parameterization: (•) real experimental data, ( $\bullet)$ simulated experimental data with REFPROP $10.0,(\bullet)$ simulated experimental data with PR EoS and MC $\alpha$ function, ( $(0)$ simulated experimental data with PR EoS and the a function with acentric factor $\dot{\omega}$, - simulated experimental data with the modified PT EoS and MC $\alpha$ function $[$ B.6]. 


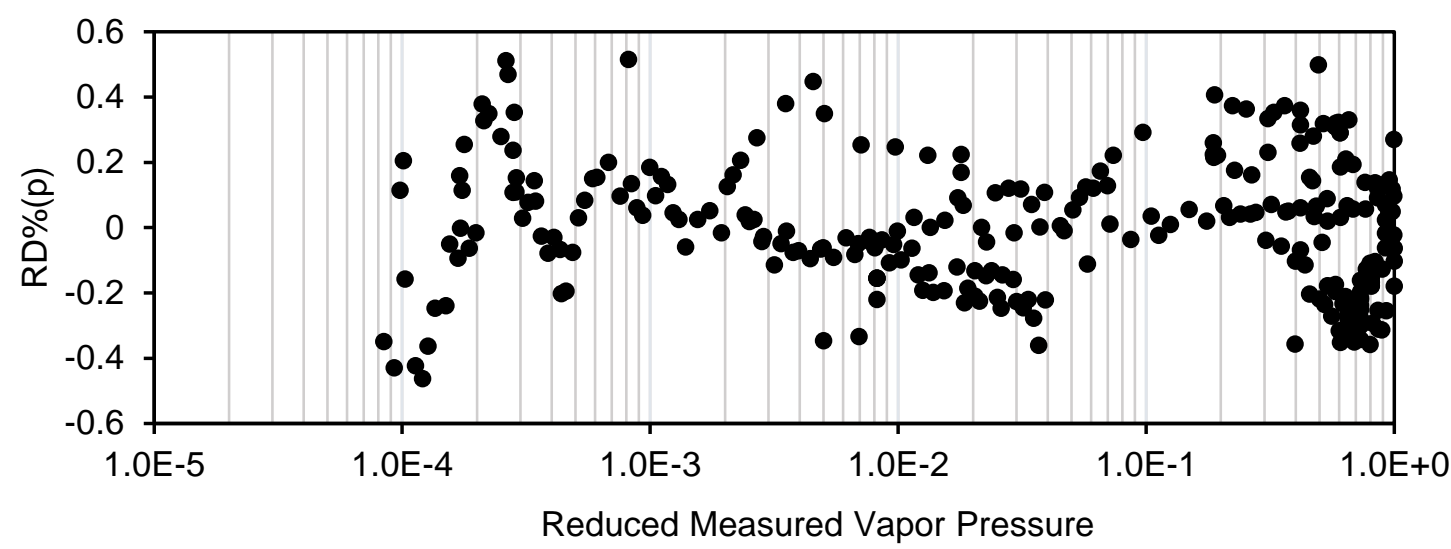

Fig E.5. Average relative deviation $\%(\overline{R D \%}(p))$ vs. reduced experimental vapor pressure for fluoromethane (R41). $\tau_{t p}=0.409 \pi_{t p}=5.87 \cdot 10^{-5}$. The evaluation is based on formulation problem (PE 3).

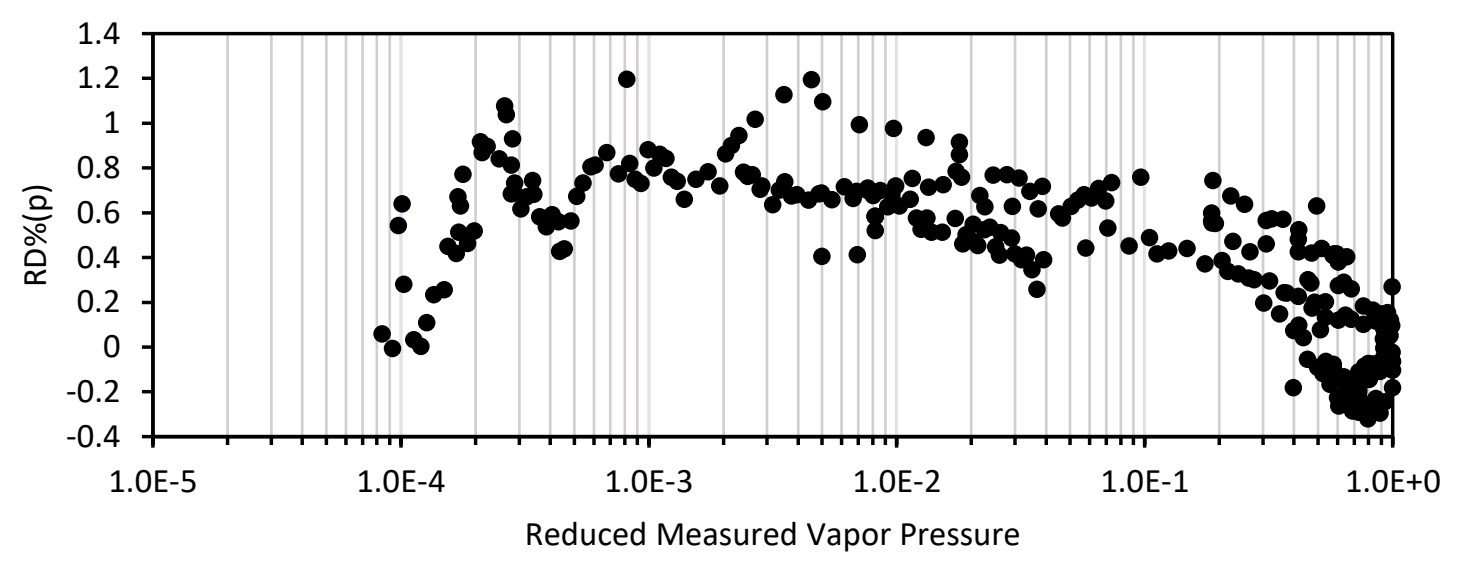

Fig. E.6. Average relative deviation $\%(\overline{R D \%}(p))$ of the predicted vapor pressures compared to the known database of vapor pressure for fluoromethane (R41). For the prediction of the vapor pressure data, vapor density data and the vapor pressure of $101325 \mathrm{~Pa}$ at the boiling temperature were used. The evaluation is based on formulation problem (PE 4) with one constraint at $\tau_{b p}=0.6138, \pi_{b p}=0.0172$. $\tau_{t p}=0.409$, $\pi_{t p}=5.87 \cdot 10^{-5}$. 


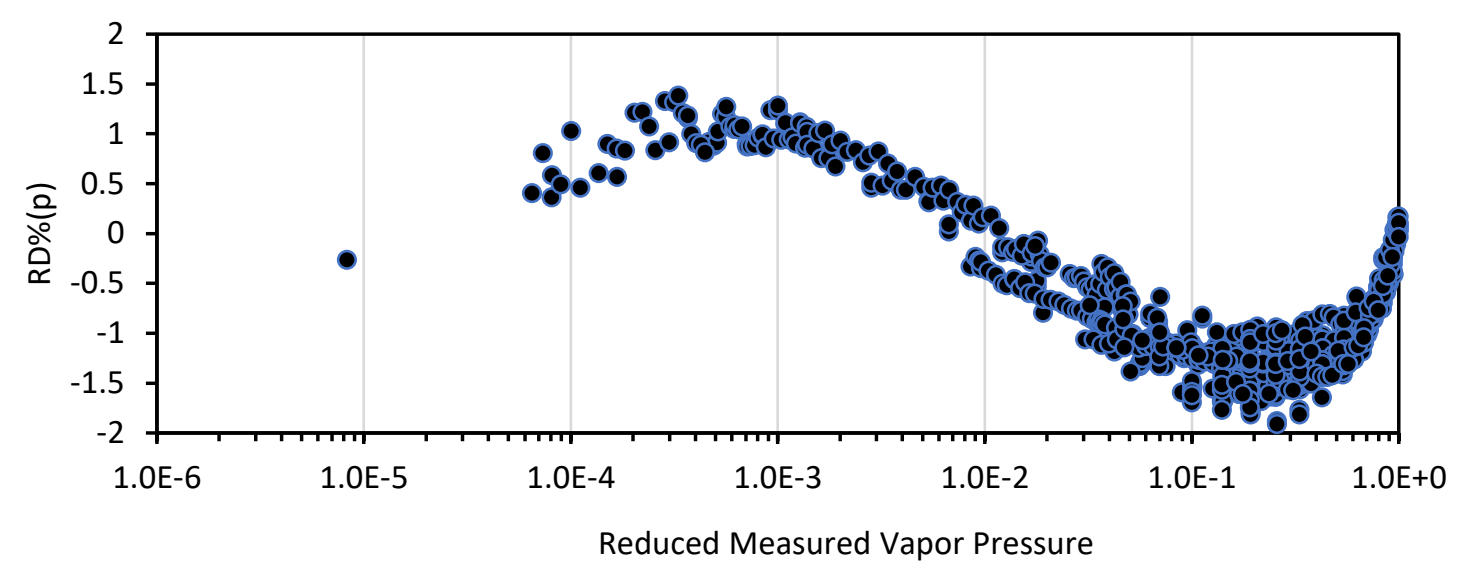

Fig. E.7. Average relative deviation $\%(\overline{R D \%}(p))$ of the predicted vapor pressures vs. reduced experimental vapor pressure for difluoromethane (R32). The predicted vapor pressure is calculated from vapor density data and only one vapor pressure data (101325 Pa) at the boiling temperature. The evaluation is based on formulation problem (PE 4) with one constraint at $\tau_{b p}=0.6303, \pi_{b p}=0.0175 . \tau_{t p}=0.388, \pi_{t p}=$ $8.29 \cdot 10^{-6}$.

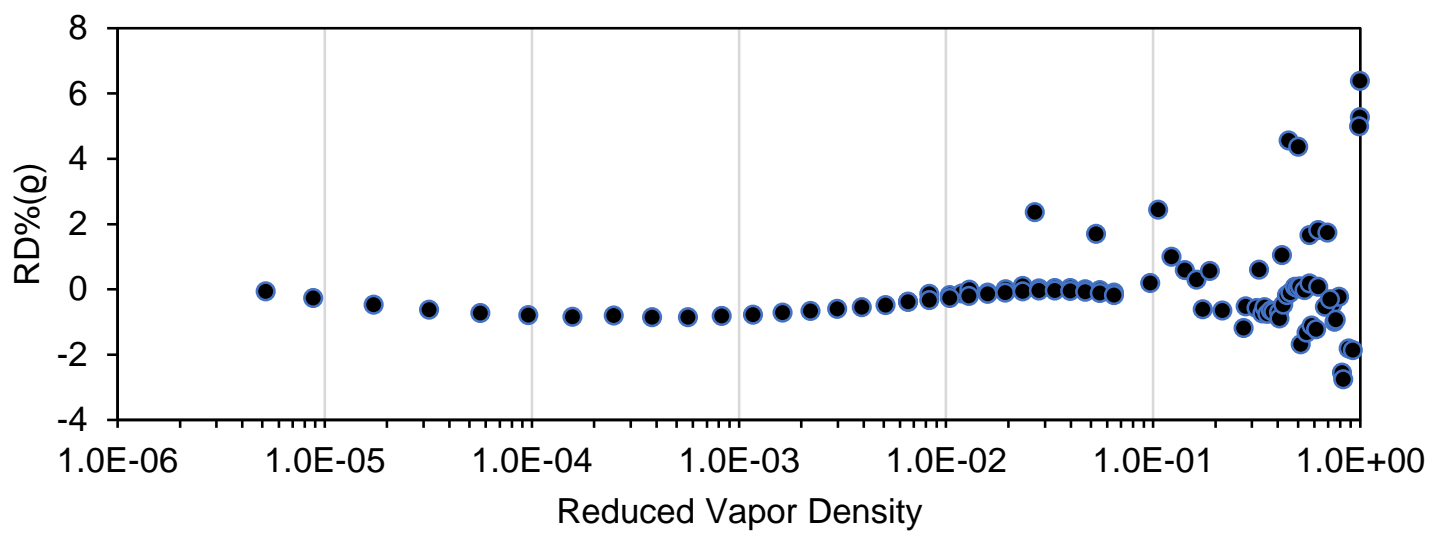

Fig. E.8. Average Relative Deviation\% $(\overline{R D \%}(\varrho))$ for vapor density of difluoromethane (R32) vs. reduced vapor density $\omega$ from the triple point to the critical temperature. $\overline{R D \%}(\varrho)$ are calculated from the semi-empirical vapor density model and density data. The density data are from experiments and calculated FEoS in REFPROP 10.0 and MBWR [B.14], see SupMat B. The evaluation is based on formulation problem (PE 3$) \cdot \omega_{t p}=$ $5.18 \cdot 10^{-6}$. 


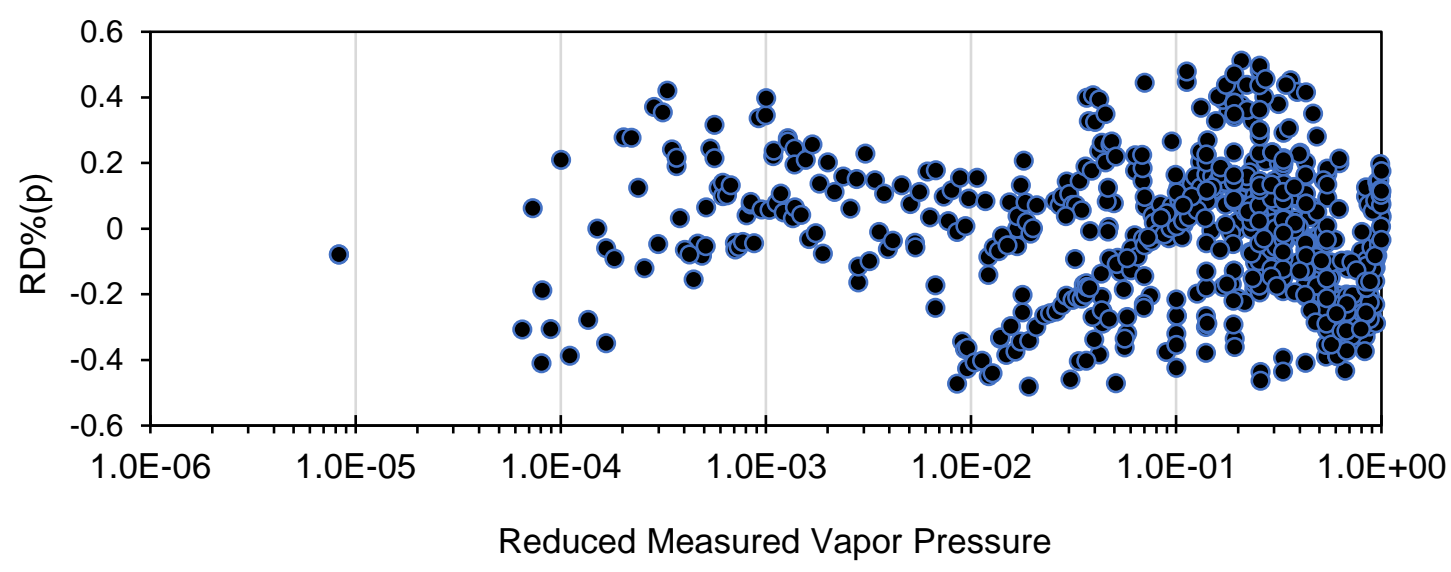

Fig. E.9. Average relative deviation $\%(\overline{R D \%}(p))$ vs. reduced measured vapor pressure for difluoromethane (R32). $\tau_{t p}=0.388, \pi_{t p}=8.29 \cdot 10^{-6}$. The evaluation is based on formulation problem (PE 3).

SupMat $F$ Why is it possible to estimate vapor pressure parameters considering vapor density data?

The derivation shall be made on the basis of the Jacobian matrix, since it is used in many Newton based parameter estimation programs. Also a direct correlation can be shown.

We start with the development of the Jacobian matrix, whose definition is given in many textbooks of mathematics on regression, such as in ref [9]. The standard deviation of the experimental data gives information about the quality of the database. Therefore, the Jacobian matrix should be developed based on the standardized residual $r\left(\tau, \bar{Y}_{Q}, \bar{S}_{Q}, \Theta\right)$ with the standard deviation $\bar{S}_{\varrho}$ of the mean $\bar{Y}_{\varrho}$ according to the equation (F.1)

$r\left(\tau, \bar{Y}_{\varrho}, \bar{S}_{\varrho}, \Theta\right)=\frac{\bar{Y}_{Q}-F_{Q}(\tau, \Theta)}{\bar{S}_{\varrho}}$.

The parameter vector $\Theta$ is defined according to Eq (33). The Jacobian matrix is then defined by Eq. (F.2) where $\bar{Y}_{\varrho}$ and $\bar{S}_{\varrho}$ are supposed to be scalar quantities.

$J\left(\tau, \bar{S}_{\varrho}, \Theta\right):=\frac{\partial r\left(\tau, \bar{Y}_{Q}, \bar{S}_{Q}, \Theta\right)}{\partial \Theta^{T}}=-\frac{1}{\bar{S}_{\varrho}} \cdot \frac{\partial F_{Q}(\tau, \Theta)}{\partial \Theta^{T}}$.

The partial derivatives for the semi-empirical vapor density model $F_{Q}(\tau, \Theta)$ are then obtained as follows (eq. (F.3 and F.4))

$$
\begin{aligned}
& G_{j}(\tau, \Theta):=\left(\frac{\partial F_{\varrho}(\tau, \Theta)}{\partial \Theta_{j}}\right)_{j=1 \ldots 6}=\frac{\varrho_{c} Z_{c} f_{p}(\tau, \Theta)}{\tau}\left(\frac{\partial Z_{M}(\tau, \Theta)^{-1}}{\partial \Theta_{j}}\right)_{j=1 \ldots 6} \\
& G_{i}(\tau, \Theta):=\left(\frac{\partial F_{Q}(\tau, \Theta)}{\partial \Theta_{i}}\right)_{i=7 \ldots 9}=\frac{\varrho_{c} Z_{c}}{\tau Z_{M}(\tau, \Theta)}\left(\frac{\partial f_{p}(\tau, \Theta)}{\partial \Theta_{i}}\right)_{i=7 \ldots 9} .
\end{aligned}
$$


The last two equations show impressively that each matrix element of the Jacobian matrix holds the vapor pressure parameters $\Theta_{i}(i=7 \ldots 9)$. This is the answer to the question of the heading for this supMat. It is the necessary but not sufficient condition that must be met for the parameters to be estimated numerically.

For this, a second condition must be satisfied in order to estimate the vapor pressure parameters as well, namely, the full rank of the variancecovariance matrix $\operatorname{cov}\left(\boldsymbol{\tau}, \overline{\boldsymbol{S}}_{\varrho}, \hat{\Theta}\right)$ of the estimated parameters $\hat{\Theta}$.

For this purpose, the Jacobian matrix of the experimental data for the individual experimental temperatures $\boldsymbol{\tau}=\left(\tau_{1} \ldots \tau_{\mathcal{N}_{\varrho}}\right)^{\mathcal{T}}$ and standard deviations $\overline{\boldsymbol{S}}_{\varrho}=$ $\left(\bar{S}_{\varrho 1} \ldots \bar{S}_{\varrho \mathcal{N}_{\varrho}}\right)^{\mathcal{T}}$ according to the equation $(\mathrm{F} .5)$ is required

$\boldsymbol{J}\left(\boldsymbol{\tau}, \overline{\boldsymbol{S}}_{\varrho}, \Theta\right)=\left(\begin{array}{ccc}\frac{G_{1}\left(\tau_{1}, \Theta\right)}{\bar{S}_{\varrho 1}} & \cdots & \frac{G_{9}\left(\tau_{1}, \Theta\right)}{\bar{S}_{\varrho 1}} \\ \vdots & \ddots & \vdots \\ \frac{G_{1}\left(\tau_{\mathcal{N}_{\Theta}, \Theta}\right)}{\bar{S}_{\varrho N_{\Theta}}} & \cdots & \frac{G_{9}\left(\tau_{\left.\mathcal{N}_{\Theta}, \Theta\right)}\right.}{\bar{S}_{Q \mathcal{N}_{\Theta}}}\end{array}\right)$.

The matrix $J\left(\boldsymbol{\tau}, \overline{\boldsymbol{S}}_{\varrho}, \Theta\right)^{\mathcal{T}} \boldsymbol{J}\left(\boldsymbol{\tau}, \overline{\boldsymbol{S}}_{\varrho}, \Theta\right)$ must have full rank before the variancecovariance matrix can be calculated according to as an inverse

$\operatorname{cov}\left(\tau, \bar{S}_{\varrho}, \widehat{\Theta}\right)=\left(J\left(\tau, \bar{S}_{\varrho}, \widehat{\Theta}\right)^{\mathcal{T}} J\left(\tau, \bar{S}_{\varrho}, \widehat{\Theta}\right)\right)^{-1}$

If full rank exists, then all parameters can be estimated. The full rank depends only on the quality of the experimental vapor density data. Thus the estimateability of the vapor pressure parameters also depends on it.

Full rank is available for fluoromethane and difluoromethane. The $\operatorname{cov}\left(\boldsymbol{\tau}, \overline{\mathbb{S}}_{\varrho}, \hat{\Theta}\right)$ matrix with the standard deviations from the Monte Carlo simulations also have full rank for both compounds.

References for the supplementary material

[A.1] Atomic Weights and Isotopic Compositions for All Elements, The NIST reference on Constants, Units and Uncertainty https://physics.nist.gov/cgibin/Compositions/stand alone.pl

[A.2] NIST Standard Reference Database 202 Web Thermo Tables (WTT). https://www.nist.gov/mml/acmd/trc/web-thermo-tables-wtt/nist-srd-wtt-202lite

[A.3] M. Frenkel, R.D. Chirico, V. Diky, X. Yan, Q. Dong, C. Muzny, ThermoData Engine (TDE): software implementation of the dynamic data evaluation concept, Journal of chemical information and modeling 45 (2005) 816-838. https://doi.org/10.1021/ci050067b 
[A.5] E.W. Lemmon, R. Span, Short Fundamental Equations of State for 20 Industrial Fluids, Journal of Chemical \& Engineering Data 51 (2006) 785-850. https://doi.org/10.1021/je050186n

[A.6] R. Tillner-Roth, A. Yokozeki, An International Standard Equation of State for Difluoromethane (R-32) for Temperatures from the Triple Point at 136.34 K to $435 \mathrm{~K}$ and Pressures up to $70 \mathrm{MPa}$, J. Phys. Chem. Ref. Data 26 (1997) 1273-1328. https://doi.org/10.1063/1.556002

[A.7] H.A. Duarte-Garza, J.W. Magee, Subatmospheric Vapor Pressures for Fluoromethane (R41), 1,1-Difluoroethane (R152a), and 1,1,1-Trifluoroethane (R143a) Evaluated from Internal-Energy Measurements, International Journal of Thermophysics 20 (1999) 1467-1481.

https://doi.org/10.1023/A:1021493106087

[A.8] J.G. Kushmerick, Fundamental Physical Constants - NIST Standard Reference Database 121 - Physical Measurement Laboratory at National Institute of Standards and Technology (NIST), 100 Bureau Drive, Gaithersburg, MD 20899, United States. 2022.

https://physics.nist.gov/cuu/Constants/Table/allascii.txt

[B.2] S.N. Biswas, C.A. Ten Seldam, S.A.R.C. Bominaar, N.J. Trappeniers, Liquid-vapor coexistence curve of methyl fluoride in the critical region. Fluid Phase Equilibria 49 (1989) 1-7. https://doi.org/10.1016/03783812(89)80001-9

[B.3] W. Cawood, H.S. Patterson, Some Physical Constants of Methyl Fluoride, and the Atomic Weight of Fluorine, J. Chem. Soc. (1932) 2180-2188. https://doi.org/10.1039/JR9320002180

[B.4] D.-Y. Peng, D.B.A Robinson, New Two-constant Equation of State. Ind. Eng. Chem. Fundam. 15 (1976) 59-64. https://doi.org/10.1021/i160057a011

[B.5]P.M. Mathias and T.W. Copeman, Extension of the Peng-Robinson Equation-of-state To Complex Mixtures: Evaluation of the Various Forms of the Local Composition Concept, Fluid Phase Equilibria 13 (1983) 91-108. https://doi.org/10.1016/0378-3812(83)80084-3

[B.6] C. Coquelet, J. El Abbadi, C. Houriez, Prediction of thermodynamic properties of refrigerant fluids with a new three-parameter cubic equation of state, International Journal of Refrigeration 69 (2016) 418-436. https://doi.org/10.1016/j.ijrefrig.2016.05.017

[B.8] C.D. Holcomb, V.G. Niesen, L.J. Van Poolen, S.L. Outcalt, Coexisting densities, vapor pressures and critical densities of refrigerants $\mathrm{R}-32$ and R-152a, at 300-385 K, Fluid Phase Equilibria 91 (1993) 145-57. https://doi.org/10.1016/0378-3812(93)85085-Z

[B.9] C. Bouchot, D. Richon, Simultaneous measurements of phase equilibrium and volumetric properties by vibrating tube densimetry: Apparatus and results involving HFC. Science et Technique du Froid, CFCs The Day After, 
Padova, 21-23 Sept.19, 2 (1994) 517-24. http://www.gbv.de/dms/tib-ubhannover/184777054.pdf

[B.10] Y. Higashi, Critical parameters for HFC134a, HFC32 and HFC125, Int. J. Refrig. 17 (1994) 524-531. https://doi.org/10.1016/0140-7007(94)90028-0

[B.11] S. Kuwabara, H. Aoyama, H. Sato, K. Watanabe, Vapor-liquid coexistence curves in the critical region and the critical temperatures and densities of difluoromethane and pentafluoroehtane. J. Chem. Eng. Data 40 (1995) 112-116. https://doi.org/10.1021/je00017a025

[B.12] D.R. Defibaugh, G. Morrison, L.A. Weber, Thermodynanmic Properties of Difluoromethane, J. Chem. Eng. Data 39 (1994) 333-340.

https://doi.org/10.1021/je00014a032

[B.13] N.C. Patel, A.S. Teja. A new cubic equation of state for fluids and fluid mixtures, Chem. Eng. Sci. 37 (1982) 463-473.

https://doi.org/10.1016/0009-2509(82)80099-7

[B.14] H.M. Roder, R.D. McCarty, A Modified Benedict Webb Rubin Equation of Sate for ParaHydrogen-II, Cryogenics Division, Institute for Basic Standards, National Bureau of Standards, NBSIR 75-814 (1975) 1-50. https://nvlpubs.nist.gov/nistpubs/Legacy/IR/nbsir75-814.pdf

[C.1] B.N. Taylor, C.E. Kuyatt, Guidelines for Evaluating an Expressing the Uncertainty of NIST Measurement Results, National Institute of Standards and Technology, NIST Technical Note 1297, 1994 Edition. https://nvlpubs.nist.gov/nistpubs/Legacy/TN/nbstechnicalnote1297.pdf

[D.1] D.W. Marquardt, An algorithm for Least Squares Estimation of non Linear Parameters, J. Soc. Ind. Appl. Math. 11 (1963) 431-441. https://doi.org/10.1137/0111030

[D.2] S. Clesse, C. Ringeval, J. Rocher, Fractal initial conditions and natural parameter values in hybrid inflation, Physical Review D 80 (2009) 123534 1-16. https://doi.org/10.1103/PhysRevD.80.123534 